\title{
The X-ray to [Ne V]3426 flux ratio: discovering heavily obscured AGN in the distant Universe
}

\author{
R. Gilli ${ }^{1}$, C. Vignali $^{2}$, M. Mignoli ${ }^{1}$, K. Iwasawa ${ }^{1,3}$, A. Comastri ${ }^{1}$, and G. Zamorani ${ }^{1}$ \\ 1 INAF - Osservatorio Astronomico di Bologna, via Ranzani 1, 40127 Bologna, Italy \\ e-mail: roberto.gilli@oabo.inaf.it \\ 2 Dipartimento di Astronomia, Università degli Studi di Bologna, via Ranzani 1, 40127 Bologna, Italy \\ 3 ICREA and Institut de Ciències del Cosmos, Universitat de Barcelona, Martí i Franquès 1, 08028 Barcelona, Spain
}

Received 11 January 2010 / Accepted 29 April 2010

\begin{abstract}
We investigate the possibility of using the ratio between the $2-10 \mathrm{keV}$ flux and the $[\mathrm{Ne} \mathrm{V}] 3426$ emission line flux $(\mathrm{X} / \mathrm{NeV})$ as a diagnostic diagram to discover heavily obscured, possibly Compton-thick active galactic nuclei (AGN) in the distant Universe. While it is on average about one order of magnitude fainter than the more commonly used [O III]5007 emission line, the [Ne V]3426 line can be observed with optical spectroscopy up to $z \sim 1.5$, whereas the [O III] 5007 line is redshifted out of the optical bands already at $z \sim 0.8$. First, we calibrated a relation between $\mathrm{X} / \mathrm{NeV}$ and the cold absorbing column density $N_{\mathrm{H}}$ using a sample of 74 bright, nearby Seyferts with both X-ray and [Ne V] data available in the literature and for which the column density is determined unambiguously. Similar to what is found for the X-ray to [O III]5007 flux ratio (X/OIII), we found that the $\mathrm{X} / \mathrm{NeV}$ ratio decreases towards high column densities, as expected if [Ne V]3426 emission is a good tracer of the AGN intrinsic power. Essentially all local Seyferts with $\mathrm{X} / \mathrm{NeV}$ values below 15 are found to be Compton-thick objects. At $\mathrm{X} / \mathrm{NeV}$ values below 100, the percentage of Compton-thick nuclei decreases to $\sim 50 \%$, but $\sim 80 \%$ of the considered sample is still absorbed with $N_{\mathrm{H}}>10^{23} \mathrm{~cm}^{-2}$. Second, we applied this diagnostic diagram to different samples of distant obscured and unobscured QSOs in the Sloan Digital Sky Survey (SDSS). SDSS blue, unobscured, type-1 QSOs in the redshift range $z=[0.1-1.5]$ indeed show X/NeV values typical of unobscured Seyfert $1 \mathrm{~s}$ in the local Universe. Conversely, SDSS type-2 QSOs at $z \sim 0.5$ classified either as Compton-thick or Compton-thin on the basis of their X/OIII ratio, would have mostly been classified in the same way based on the $\mathrm{X} / \mathrm{NeV}$ ratio. We applied the $\mathrm{X} / \mathrm{NeV}$ diagnostic diagram to 9 SDSS obscured QSOs in the redshift range $z=[0.85-1.31]$, selected by means of their prominent [Ne V]3426 line (rest $E W>4 \AA$ ) and observed with Chandra ACIS-S for 10ks each ( 8 of them as part of our proprietary program). Based on the $\mathrm{X} / \mathrm{NeV}$ ratio, complemented by X-ray spectral analysis, 2 objects appear good Compton-thick QSO candidates, 4 objects appear as Compton-thin QSOs, while 3 have an ambiguous classification. When excluding broad-lined QSOs with a red continuum from the sample and thus considering only genuine narrow-line objects, the efficiency in selecting Compton-thick QSOs through the [Ne V] line is about $50 \%$ (with large errors, though), more similar to what is achieved with [O III] selection. We discuss the possibility of applying the $\mathrm{X} / \mathrm{NeV}$ diagnostic to deep X-ray surveys to search for Compton-thick Seyferts at $z \sim 1$, i.e., those objects that are thought to be responsible for the "missing" X-ray background. Finally, we compared the optical spectral properties of [Ne V]-selected QSOs with those of other SDSS populations of obscured and unobscured QSOs. By restricting the analysis to objects in the same redshift (and luminosity) range $z=[0.4-1.5]$, we found evidence that, at any given [Ne V] luminosity, increasing obscuration is accompanied by increasing [O II] 3727 emission. This correlation is interpreted as evidence of enhanced star formation in obscured QSOs, which is consistent with current popular scenarios of BH-galaxy coevolution.
\end{abstract}

Key words. galaxies: active $-\mathrm{X}$-rays: general $-\mathrm{X}$-rays: galaxies

\section{Introduction}

While the cosmological evolution of unobscured QSOs has been traced up to $z \sim 6$, the evolution of obscured AGN is much more uncertain and is the subject of intense debate. The observed number statistics in current AGN samples is dominated by unobscured objects that are easier to discover (e.g. $>10^{5}$ QSOs have been identified in the Sloan Digital Sky Survey), but several arguments suggest that obscured AGN must be intrinsically more numerous. Deep X-ray surveys (see Brandt \& Hasinger 2005 , for a review) have indeed shown that, towards faint $\mathrm{X}$ ray fluxes, the surface density of obscured AGN overtakes that of unobscured AGN. Also, population synthesis models of the cosmic X-ray background (XRB), suggest that obscured AGN outnumber unobscured ones by a factor that ranges from $\sim 2$ to $\sim 8$, depending on the considered luminosity regime (Gilli et al. 2007; see Treister et al. 2009b; and Ballantyne et al. 2006, for a steeper luminosity dependence). To understand the cosmological history of accretion onto supermassive black holes (SMBHs), it is therefore necessary to map and understand the population of obscured AGN and, in particular, of the most obscured and hence elusive ones, the so-called Compton-thick (CT) nuclei, i.e. those obscured by column densities above $\sim 10^{24} \mathrm{~cm}^{-2}$.

The population of moderately obscured AGN in fact does not completely account for the XRB peak intensity at $30 \mathrm{keV}$, to which CT AGN are expected to contribute from $\sim 10 \%$ (Treister et al. 2009b) to $\sim 25-30 \%$ (Gilli et al. 2007), depending on the XRB model assumptions. In addition, the presence of a large population of CT AGN across the cosmic epochs would help in reconciling the measured mass function of local SMBHs with 
what is expected by integrating the accretion history of seed black holes (Marconi et al. 2004; Shankar et al. 2004). Finally, popular semi-analytic models of galaxy formation and evolution (Kauffmann \& Haehnelt 2000; Marulli et al. 2008), coupled to hydrodynamical simulations of galaxy mergers (Hopkins et al. 2006), propose that nuclear activity is triggered during major mergers of gas-rich galaxies and that in its early stages, the AGN is embedded within optically thick gas shrouds. Despite this theoretical progress, the cosmological evolution and luminosity function of CT objects is currently unknown, and in the synthesis models of the XRB, it has been usually assumed to be equal to that of less obscured objects. Whether this is the case or not can only be determined by obtaining statistically significant samples of distant CT objects and by comparing them with the local samples (see e.g. Comastri 2004 and Della Ceca et al. 2008, for reviews on nearby, bona-fide CT objects).

The number of techniques devised to select CT AGN is rapidly growing, following the technological development of efficient detectors across the electromagnetic spectrum. These diverse selection techniques have allowed the first estimates of the space density of CT AGN in different redshift and luminosity regimes.

Very hard X-ray selection, i.e. at energies above $10 \mathrm{keV}$, is unaffected by absorption up to a few $\times 10^{24} \mathrm{~cm}^{-2}$, but, because of the still limited instrumental sensitivity, it mainly samples the nearby Universe. The population of CT AGN detected by INTEGRAL/IBIS (Tueller et al. 2008) and Swift/BAT (Malizia et al. 2009) at $z<0.02$ is indeed producing only a tiny fraction $(<1 \%)$ of the cosmic XRB.

Deep X-ray surveys in the more accessible $2-10 \mathrm{keV}$ band reveal large populations of heavily obscured objects in the redshift range $z \sim[0.5-4]$. The generally low photon statistics of the detected sources, however, prevent an accurate spectral analysis and $N_{\mathrm{H}}$ measurement. The CT nature of a faint X-ray source is therefore often inferred from the characteristics of the reprocessed spectrum, like the presence of a prominent fluorescence $\mathrm{K} \alpha$ iron line over a flat continuum. Examples of distant CT AGN selected with this technique have been found by Tozzi et al. (2006) and Georgantopoulos et al. $(2007,2009)$ in the Chandra Deep Fields. The number of CT AGN candidates detected in the deep X-ray surveys appears to be in rather good agreement with the XRB models predictions.

A very recent and promising approach to select CT candidates in the distant Universe is based on their strong mid-IR flux, where most of the absorbed radiation should be re-emitted (Martínez-Sansigre et al. 2005). Recently, Daddi et al. (2007), Fiore et al. (2008, 2009), and Alexander et al. (2008) have located heavily obscured AGN in objects showing $24 \mu \mathrm{m}$ emission in excess of what is expected from dust heated by stellar processes. By stacking the Chandra data of these mid-IR-excess objects, a very hard X-ray spectrum was observed, reminiscent of CT obscuration. These studies span a broad AGN luminosity range $\left(L_{\mathrm{X}} \sim 10^{42-45} \mathrm{erg} \mathrm{s}^{-1}\right)$, but mostly sample populations of objects at $z \sim 2$. The measured space density of CT AGN at these high redshifts is in general as high as expected from XRB synthesis models or possibly even higher (see e.g. Treister et al. 2009a).

Another way to select obscured QSOs is through their highionization narrow optical emission lines, which are thought to be produced on physical scales (from $\sim 0.1$ to a few kpc) mostly free of nuclear obscuration. Recently, the [O IV] $26 \mu \mathrm{m}$ line has been used to select obscured AGN among galaxies observed with Spitzer/IRS. However, since this line quickly moves out of the observable IR bands as redshift increases, this selection mostly concerns the nearby Universe (Diamond-Stanic et al. 2009; Rigby et al. 2009). The most commonly used marker of obscured nuclear activity therefore remains the [O III]5007 emission line, which is strong, falls in the optical domain, and allows object selection up to $z \sim 0.8$.

The $2-10 \mathrm{keV}$ to $[\mathrm{O}$ III]5007 flux ratio (X/OIII) has often been used as a diagnostic for heavy obscuration in sources with poor X-ray photon statistics (Maiolino et al. 1998; Cappi et al. 2006; Panessa et al. 2006): low X/OIII ratios ( $\$ 3$, see e.g. Fig. 4 of Cappi et al. 2006) are indeed highly suggestive of heavy nuclear absorption. Based on the [O III]5007 emission line, Zakamska et al. (2003) and Reyes et al. (2008) have identified a population of obscured QSOs in the Sloan Digital Sky Survey (SDSS) at a median redshift of $z \sim 0.3$, at least as abundant as that of type-1 QSOs at the same redshifts (Reyes et al. 2008). These results have been extended to lower luminosities by Bongiorno et al. (2010), who measured the luminosity function of [O III]-selected type-2 AGN in the zCOSMOS spectroscopic survey (Lilly et al. 2007), finding that the fraction of obscured AGN is decreasing with luminosity, in agreement with what is observed in X-ray surveys.

X-ray observations of small samples drawn from the Zakamska et al. (2003) catalog suggest that about half of luminous type-2 QSOs $\left(\log L_{\mathrm{OIII}}>9.3 L_{\odot}\right)$ could be CT (Ptak et al. 2006; Vignali et al. 2006, 2010, hereafter V10; see also Lamastra et al. 2009, for X-ray observations of lower luminosity SDSS type-2 AGN). Since [O III] selection is likely missing objects in which the narrow line region (NLR) is also extincted (as in the prototype CT AGN NGC 4945 and NGC 6240), the estimated type-2 QSO abundances should be considered as lower limits (Reyes et al. 2008, V10).

In this work we explore the possibility of using the high-ionization $[\mathrm{Ne}$ V]3426 emission line, rather than the [O III]5007 line, as a tracer of obscured nuclear activity. Despite being on average a factor of $\sim 9$ weaker than [O III]5007 (Ferland \& Osterbrock 1986; Zakamska et al. 2003) and suffering stronger dust extinction, the $[\mathrm{Ne} \mathrm{V}] 3426$ line is commonly observed in nearby Seyfert galaxies and, given that high-energy photons $(\gtrsim 0.1 \mathrm{keV})$ are required to further ionize NeIV, it is considered an unambiguous sign of nuclear activity (e.g. Schmidt et al. 1998). In addition, the [Ne V]3426 emission line is observable up to $z \sim 1.5$ before being redshifted out of the optical bands, whereas the [O III]5007 line is only observable up to $z \sim 0.7-0.8$. Indeed, only 13 out of 887 [O III]-selected QSOs in the Reyes et al. (2008) sample lie at $z>0.7$, with only 3 at $z>0.8$. Then, [Ne V]-selection may be used to reveal nuclear activity in obscured sources at $z \sim 1$, i.e. at the epoch where most of the XRB light is thought to be produced.

The structure of the paper is the following. In Sect. 2 we present and discuss the sample of nearby Seyfert galaxies used to calibrate the relation between $[\mathrm{Ne} \mathrm{V}]$ and X-ray emission (the details of the sample are given in the Appendix). In Sect. 3 we present the $\mathrm{X} / \mathrm{NeV}$ diagnostic diagram and apply it to obscured and unobscured QSO populations drawn from the SDSS. In Sect. 4 we present Chandra observations of a sample of $9[\mathrm{Ne} \mathrm{V}]$-selected obscured QSOs at $z \sim 1$ in the SDSS and use the $\mathrm{X} / \mathrm{NeV}$ diagnostic to estimate the fraction of $\mathrm{CT}$ objects among them. In Sect. 5 we discuss efficiency and biases of [Ne V] selection, together with its application to sky areas with deep optical spectroscopy and X-ray coverage. In the same section, the evidence of enhanced star formation in obscured QSOs at $z=0.4-1.5$ is also highlighted. The conclusions are drawn in Sect. 6. 


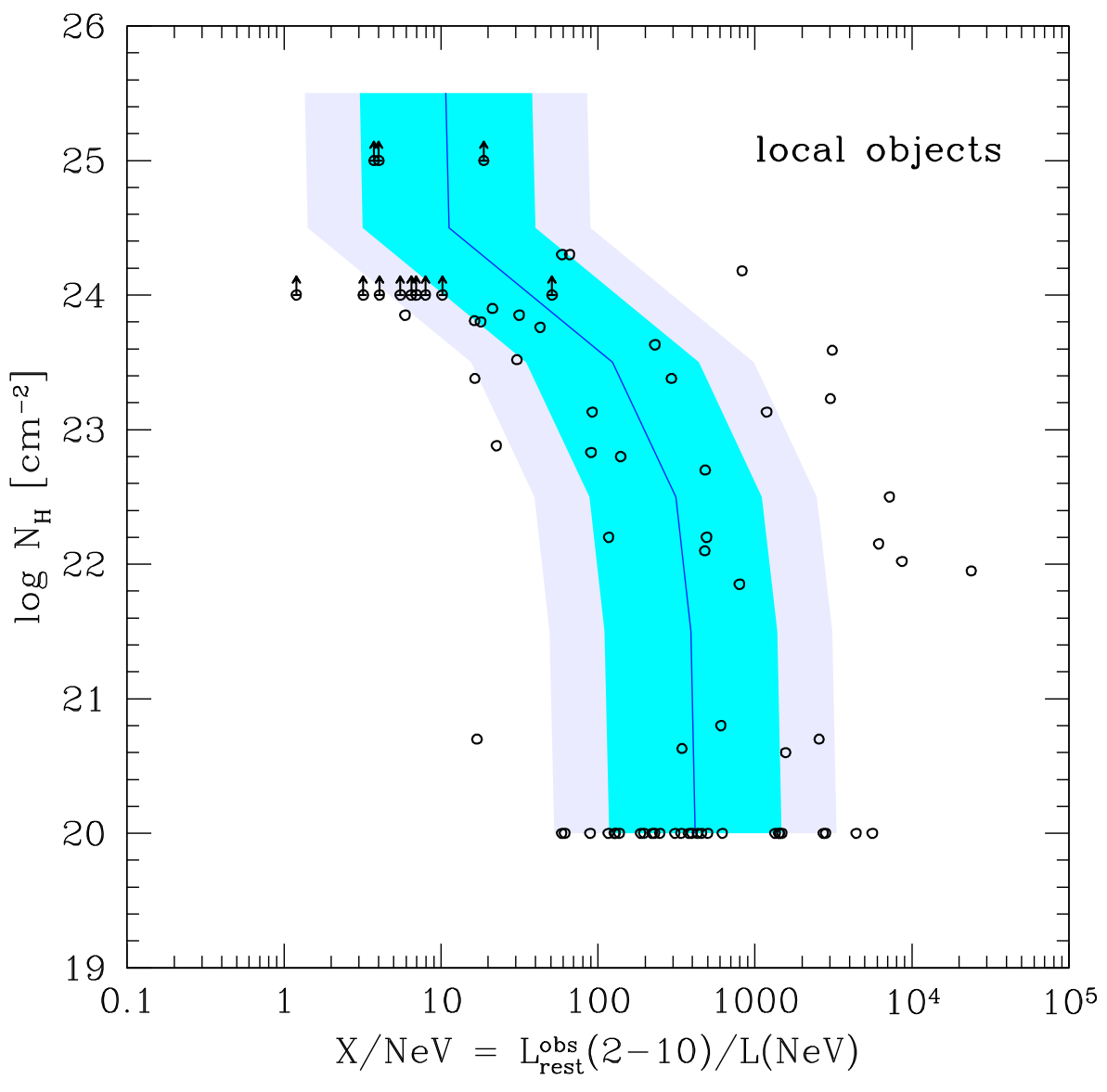

Fig. 1. Observed 2-10 keV to [Ne V]3426 luminosity ratio $(\mathrm{X} / \mathrm{NeV})$ vs. absorption column density for a sample of 74 Seyfert galaxies in the local Universe. The solid line shows the expected trend obtained by starting from the mean $\mathrm{X} / \mathrm{NeV}$ ratio $\langle\mathrm{X} / \mathrm{NeV}\rangle$ observed in unobscured objects (i.e. those plotted at $\log N_{\mathrm{H}}=20$ ) and progressively obscuring the $\mathrm{X}$-ray emission with increasing $N_{\mathrm{H}}$ (up to $\log N_{\mathrm{H}}=25.5$ ) as plotted on the $y$-axis. Lower $\operatorname{limits}$ at $\log N_{\mathrm{H}}=$ 24 refer to Compton-thick (CT) objects observed only below $10 \mathrm{keV}$, while datapoints at $\log N_{\mathrm{H}}>24$ and lower limits at $\log N_{\mathrm{H}}=25$ refer to CT objects observed also above $10 \mathrm{keV}$, for which a more stringent determination of the column density is possible. The darker (lighter) shaded region is obtained by making the same computation as above but starting at $\pm 1 \sigma( \pm 90 \%)$ around $\langle\mathrm{X} / \mathrm{NeV}\rangle$ (see text for details). The region at low $(<15) \mathrm{X} / \mathrm{NeV}$ values is essentially populated only by CT objects.

\section{The local Seyfert sample}

We searched in the literature for nearby $(z<0.1)$ AGN for which both $[\mathrm{Ne} \mathrm{V}]$ and X-ray data are available. A total sample of 74 objects were found with measured [Ne V] flux, 2-10 keV flux, and X-ray column density $N_{\mathrm{H}}$. The most difficult information to obtain was that on [Ne V] flux, since optical spectra are often limited at wavelengths $>3700 \AA$. In contrast, the $N_{\mathrm{H}}$ and the $2-10 \mathrm{keV}$ flux values for bright nearby objects are more easily obtained either from published papers or from archival X-ray data. The main catalogs of optical/near-UV Seyfert spectra providing the $[\mathrm{Ne} \mathrm{V}]$ fluxes used in this work are those published by Malkan (1986), Morris \& Ward (1988), Storchi-Bergmann et al. (1995), Erkens et al. (1997), as well as the compilation by Schmitt (1998). A public catalog of HST/STIS spectra of nearby AGN has been released by Spinelli et al. (2006). The HST/STIS spectra have been extracted in apertures of $0.2^{\prime \prime} \times 0.2^{\prime \prime}$, thus sampling physical scales of a few tens of parsecs at the typical source redshift. This physical scale is often too small to fully encompass the NLR, which indeed may extend up to a few kpc. We verified that the $[\mathrm{O} \mathrm{III}]$ and $[\mathrm{Ne} \mathrm{V}]$ fluxes as measured on the Spinelli et al. (2006) spectra are often significantly lower (an order of magnitude or more) than those measured on larger apertures, especially in those objects in which an extended NLR has been revealed (Bianchi et al. 2006). Therefore, we avoided using measurements obtained with HST/STIS and considered only those taken with larger apertures. All the [Ne V] fluxes used in this work come from apertures $\gtrsim 1.5^{\prime \prime}$. Whenever more than one measurement is available for the [Ne V] flux, we preferred the one obtained with the largest aperture. For 6 objects we measured the $[\mathrm{Ne} \mathrm{V}]$ line flux directly on the calibrated spectrum drawn from the catalog of 99 UV-optical spectra of nearby galaxies released by Storchi-Bergmann et al. ${ }^{1}$.

Since our main aim is to calibrate a diagnostic that can be applied to distant objects, we did not attempt to correct the $[\mathrm{Ne} \mathrm{V}]$ fluxes for the reddening that may be intrinsic to the NLR. Indeed, while for local objects the extinction to the NLR can be easily measured through e.g. the ratio between the narrow components of $\mathrm{H} \alpha$ and $\mathrm{H} \beta$, in distant AGN either $\mathrm{H} \alpha$ or both $\mathrm{H} \alpha$ and $\mathrm{H} \beta$ are not observable and measuring the ratio between the Balmer lines of higher order (e.g. $\mathrm{H} \beta / \mathrm{H} \gamma, \mathrm{H} \gamma / \mathrm{H} \delta$ ) is often unfeasible due to their weakness over noisy spectra.

As for X-ray data, we generally preferred to use the values obtained with the most recent and sensitive satellites Chandra and XMM-Newton. When dealing with heavily obscured objects, though, we considered data obtained with Suzaku and BeppoSAX in order to map the energy range above $10 \mathrm{keV}$ and get either a measurement or a tighter lower limit on the absorbing column (see Fig. 1). Sometimes an observed 2-10 keV flux measurement is not directly quoted in the considered literature papers: in those cases we estimated it using the published best-fit spectral parameters and/or 2-10 keV luminosity. For a few objects we analyzed archival X-ray data that were still unpublished. The full sample, along with [Ne V] fluxes, 2-10 keV fluxes, $N_{\mathrm{H}}$ values, and relative references is presented in the Appendix.

\subsection{Sample biases}

Although an effort was made to find as many sources as possible with both [ $\mathrm{Ne} \mathrm{V}]$ and $\mathrm{X}$-ray information, the sample we

${ }^{1}$ http://www.if.ufrgs.br/ thaisa/ 
built is by no means complete and cannot be considered suitable for statistical studies. Objects in the sample were targeted for observations for several different reasons. Moreover, since we require $[\mathrm{Ne} \mathrm{V}]$ detection, the sample might be biased towards objects with stronger [ $\mathrm{Ne} \mathrm{V}]$ emission. Indeed, the absence of a $[\mathrm{Ne} \mathrm{V}]$ measurement may well be due to the lack of spectral coverage at $3426 \AA$, but also to excessively faint [Ne V] emission. Given that sometimes the information on the spectral coverage is missing and that not all optical spectra on which line measurements are derived have been published, it is often difficult to judge what the reason is for the lack of [Ne V] data. In contrast, whenever a source is observed in X-ray, the fine sensivity of current X-observatories and the brightness of the considered nearby objects generally allow a measurement of both $2-10 \mathrm{keV}$ flux and column density. Therefore, the sample should not be biased towards X-ray bright objects ${ }^{2}$. Also, those objects in which the NLR is relatively free of obscuration should appear mainly in the sample. Indeed, even a modest reddening of $E(B-V)=0.5$ corresponds to a flux dumping by a factor of $\sim 10$ at $3426 \AA$ assuming standard extinction curves. Compton-thick objects like NGC 4945 and NGC 6240, in which the NLR is affected by strong extinction, indeed do not appear in the sample. Because of these issues, the present sample is the one to use when building a diagnostic diagram to be applied to samples of distant sources selected on the basis of their [Ne V] emission, which indeed would suffer from similar biases.

\section{The $\mathrm{X} / \mathrm{NeV}$ diagnostic}

We computed the ratio between the fluxes observed in the $2-10 \mathrm{keV}$ band and in the $[\mathrm{Ne} \mathrm{V}] 3426$ emission line $(\mathrm{X} / \mathrm{NeV})$ for the local Seyfert sample described in the previous section and plotted the $\mathrm{X} / \mathrm{NeV}$ value against the measured $\mathrm{X}$-ray absorption (Fig. 1). This is essentially the same diagram as worked out by Maiolino et al. (1998; see also Cappi et al. 2006; and Panessa et al. 2006) obtained by replacing the [O III] with the [Ne V] line. Working with local objects allows dealing with good optical and $\mathrm{X}$-ray spectra and in turn provides a precise measurement of the $\mathrm{X}$-ray absorption. Assuming that the [Ne V]3426 flux is mostly produced in the NLR, far from the nuclear obscuration that affects the X-ray emission, one would expect that the $\mathrm{X} / \mathrm{NeV}$ ratio on average decreases with increasing column density. This is indeed what is observed (see Fig. 1): the median $\mathrm{X} / \mathrm{NeV}$ values for unabsorbed and for CT Seyferts are about 400 and 7, respectively. For $\mathrm{X} / \mathrm{NeV}<15$, almost all objects are CT. For $\mathrm{X} / \mathrm{NeV}<100$, about $50 \%$ of the objects are CT, while $80 \%$ are still obscured by columns over $10^{23} \mathrm{~cm}^{-2}$. The CT outlier with $\mathrm{X} / \mathrm{NeV} \sim 800$, NGC 3281, likely suffers from extinction in the NLR. NGC 6240 and NGC 4945 do not even appear in the diagram. Therefore, low $\mathrm{X} / \mathrm{NeV}$ ratios would select clean samples of CT AGN, i.e. not significantly contaminated by lowobscuration sources, but not complete.

For unabsorbed Seyferts, the mean and median $\mathrm{X} / \mathrm{NeV}$ values are almost identical and very similar to what is obtained by scaling down the average X/OIII value obtained by Panessa et al. (2006) for Seyfert 1 s by a factor of 9, i.e., the average

\footnotetext{
${ }^{2}$ We only found very few local objects with [ $\left.\mathrm{Ne} \mathrm{V}\right]$ emission and observed in X-ray, which show poor X-ray photon statistics, despite having similar X-ray fluxes to those analyzed in the previous section. These objects were observed with very short exposures (e.g. by the XMMSlew survey) and have not been considered here since it is not possible to have reliable information on their column density and hence place them in Fig. 1.
}

OIII/NeV emission line ratio measured for local Seyferts (e.g. Shuder \& Osterbrock 1981). In Fig. 1 we show the $\mathrm{X} / \mathrm{NeV}$ vs. column density relation obtained by starting from the mean $\mathrm{X} / \mathrm{NeV}$ ratio observed in unobscured Seyfert 1s and progressively absorbing the $2-10 \mathrm{keV}$ flux of unobscured AGN using the spectral templates of Gilli et al. (2007). In particular, the 2-10 keV emission in CT AGN with $\log N_{\mathrm{H}}>24.5$, mostly dominated by the Compton-reflected continuum, was assumed to be about $\sim 2 \%$ of the intrinsic one in the same band. The $1 \sigma$ and $90 \%$ limits of the $\mathrm{X} / \mathrm{NeV}$ vs. $N_{\mathrm{H}}$ relation are also shown in Fig. 1. Most objects lie within the $90 \%$ limits, in agreement with the expectations that nuclear absorption affects only the X-ray emission. However there are a few notable exceptions. For instance, the object with the highest X/NeV ratio in Fig. 1 is NGC 2992, a Seyfert 1.9 galaxy in which the X-ray flux has been observed to vary by a factor of 20 over the years, probably as a consequence of switching its nuclear activity on and off (Gilli et al. 2000) and/or because of flares in the inner accretion disk (Murphy et al. 2007). Using the average X-ray flux, rather than the highstate X-ray flux as done in Fig. 1, would shift NGC 2992 in the shaded area where most of the sources lie. This highlights the problem of dealing with variable sources and non-simultaneous optical and X-ray observations, which increases the dispersion of the $\mathrm{X} / \mathrm{NeV}$ distribution. Absorption variations are also important. Indeed, the lightly obscured object $\left(\log N_{\mathrm{H}}<21\right)$ with the lowest $\mathrm{X} / \mathrm{NeV}$ ratio $(\mathrm{X} / \mathrm{NeV} \sim 20)$ is the dwarf AGN POX 52 (Barth et al. 2004), which shows rapid X-ray flux and absorption variability (Thornton et al. 2008). Based on the observed variability, the position of POX 52 on the diagram can shift from the plotted position to $\mathrm{X} / \mathrm{NeV} \sim 10$ and $\log N_{\mathrm{H}}=22.8$, in the region populated by obscured objects. Finally, it is worth noting that in radio-loud objects an additional $\mathrm{X}$-ray component coming from the jet emission, produces a shift towards higher $\mathrm{X} / \mathrm{NeV}$ ratios than radio-quiet objects: indeed $\sim 90 \%$ of the radio galaxies in our samples show $\mathrm{X} / \mathrm{NeV}$ ratios over 400 , including some of the objects falling on the right of the shaded region in Fig. 1, while the same fraction is $\sim 25 \%$ for the radio-quiet galaxies.

\subsection{SDSS type-1 QSOs}

We applied the $\mathrm{X} / \mathrm{NeV}$ diagnostic ratio derived in the local Universe to distant obscured and unobscured QSOs in the SDSS. What is needed here is the $2-10 \mathrm{keV}$ rest frame flux (or luminosity) prior to absorption correction, to make a meaningful comparison with the local sample. We first considered the catalog of Young et al. (2009a, hereafter Y09), who collected a sample of 792 QSOs in the SDSS DR5 selected primarily by their blue optical colors and serendipitously observed by XMM-Newton ${ }^{3}$. By matching the Y09 sample with the SDSS spectroscopic tables we found 94 objects in the redshift range $z=[0.12-1.50]$ for which i) the [Ne V]3426 emission line is detected at $>3 \sigma$ and ii) have been detected in the X-rays at $>6 \sigma$, which is the threshold adopted by Y09 to perform X-ray spectral fits and measure both the observed $2-10 \mathrm{keV}$ rest frame flux and the column density $N_{\mathrm{H}}$. To check for possible problems in the determination of the [Ne V]3426 flux related to the SDSS automatic procedures, we visually inspected the 94 SDSS spectra above and found 11 objects in which the listed detection of [ $\mathrm{Ne} \mathrm{V}]$ emission is seriously affected by instrumental features and sky residuals. These objects were then removed from the sample. We also noticed that the line parameters listed in the SDSS spectroscopic

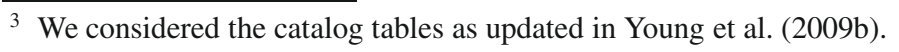




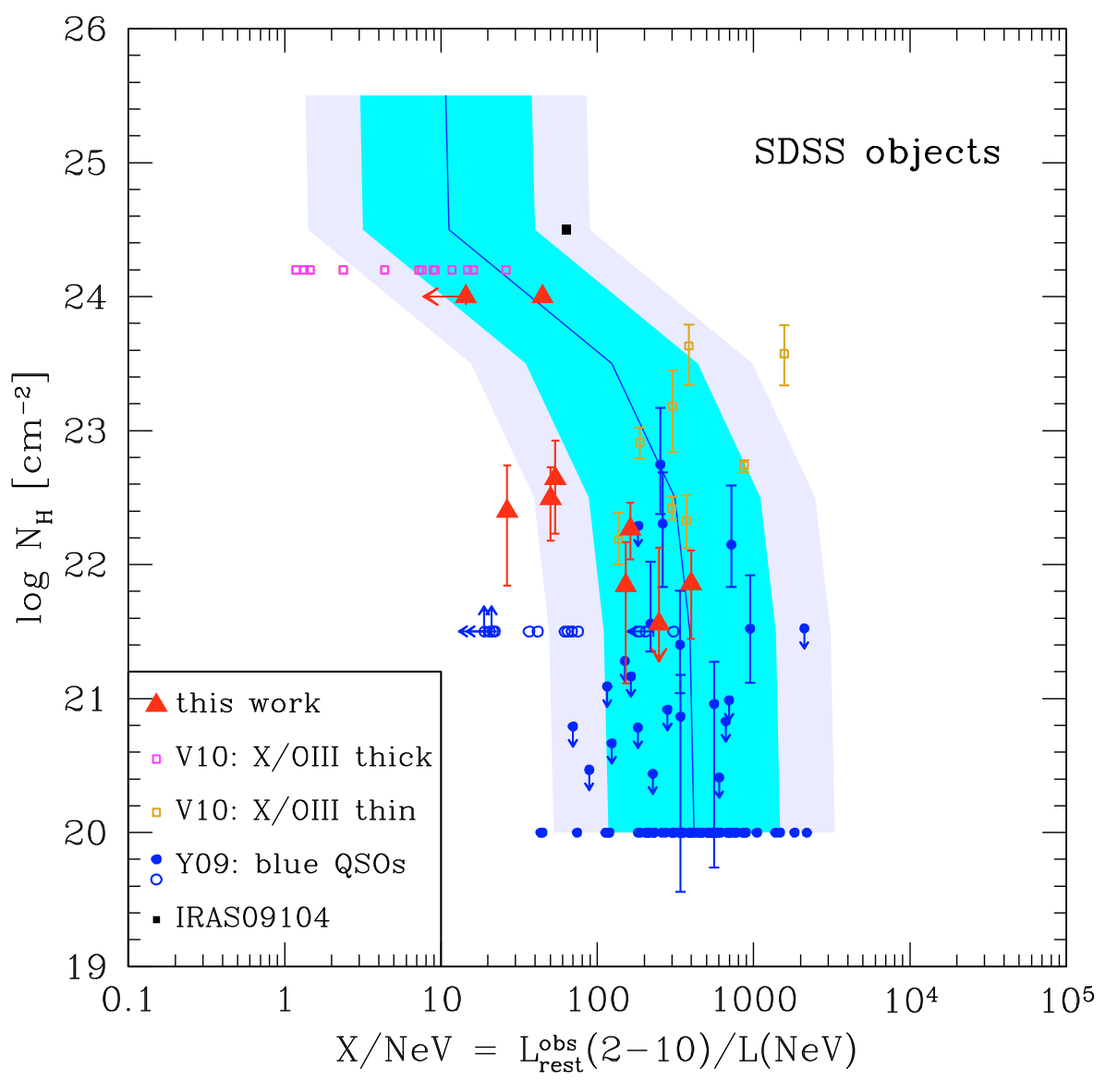

Fig. 2. The $\mathrm{X} / \mathrm{NeV}$ vs. $N_{\mathrm{H}}$ diagram (see Fig. 1) applied to different samples of SDSS QSOs. The blue SDSS QSOs in the Young et al. (2009a, Y09) catalog populate the same region of unobscured Seyfert 1s in the local Universe: filled circles are high-significance $\mathrm{X}$ ray detections for which the $N_{\mathrm{H}}$ values have been measured from the spectral fits by Y09; open circles are low-significance or non X-ray detections in Y09, which have been plotted at $\log N_{\mathrm{H}}=21.5$ (see Sect. 3.1 for details). The obscured SDSS QSOs in the V10 sample are plotted as open squares (CT candidates are placed at $\log N_{\mathrm{H}}=24.2$ ). Objects which have been classified as Compton-thick (-thin) by V10 on the basis of their low (high) $\mathrm{X} / \mathrm{OIII}$ ratio, show correspondingly low (high) $\mathrm{X} / \mathrm{NeV}$ ratios, hence would also be classified as Compton-thick (-thin) based on this new diagnostic. The [Ne V]-selected obscured QSOs investigated in this work are shown as filled triangles. The two objects classified in Table 3 as likely CT QSO candidates based on their $\mathrm{X} / \mathrm{NeV}$ ratio and/or $\mathrm{X}$-ray spectral properties, are plotted at $\log N_{\mathrm{H}}=24$ (see also Sect. 4.2). For comparison, the position of the SDSSobserved, CT QSO candidate IRAS 09104 is also shown with a filled square. tables are not accurate for weak emission lines over a steep continuum. For instance, in about one quarter of SDSS QSOs, the [O II]3727 emission line (see the Discussion) appears as an absorption line because the continuum is overestimated. We then retrieved the SDSS spectra and performed a Gaussian fit to the emission lines we were interested in, thereby deriving fluxes, luminosities, and rest-frame equivalent widths. All the emission line parameters used in this work for SDSS objects are derived from our direct fits. Whenever we compared our line measurements with those performed by other authors on the same SDSS spectra (e.g. for a subsample of type-2 QSOs in Zakamska et al. 2003), we found excellent agreement.

The $\mathrm{X} / \mathrm{NeV}$ vs. $N_{\mathrm{H}}$ relation for the 83 SDSS QSOs selected above is shown in Fig. 2. Most objects are unobscured and populate the same region of local Seyfert 1 galaxies. The median $\mathrm{X} / \mathrm{NeV}$ ratio for SDSS QSOs is 370, almost identical to the value of 400 found for local Seyfert 1s. The few objects showing significant intrinsic X-ray absorption appear in the optical as intermediate type Seyferts (i.e. 1.8-1.9) rather than pure type-1 $\mathrm{AGN}^{4}$.

To check for possible outliers, e.g. blue SDSS QSOs with very low $\mathrm{X} / \mathrm{NeV}$ ratios $(<15$, as observed for local CT Seyferts), we also considered those objects in the Y09 catalog that show significant $[\mathrm{Ne} \mathrm{V}]$ emission and that were either just detected (13 objects) or completely undetected (3 objects) in X-ray. We verified that only two objects - appearing in the optical as classical, blue, broad line objects - based on the [Ne V] flux we measured and on the $2-10 \mathrm{keV}$ rest frame flux in the

\footnotetext{
${ }^{4}$ One of them is indeed also included in the type-2 QSOs catalog by Zakamska et al. (2003) and in the Vignali et al. (2006, 2010) samples.
}

Y09 catalog, would nominally show $\mathrm{X} / \mathrm{NeV}<15$. We doublechecked the $\mathrm{X}$-ray fluxes of these sources based on literature data (i.e. a Chandra $50 \mathrm{ks}$ exposure available for one of them, providing a good quality spectrum with 200 photons, and the 2XMM catalog results for the other source) and found that, while the soft X-ray fluxes agree with the Y09 values, the $2-10 \mathrm{keV}$ fluxes appear significantly (i.e. a factor of 5-10) higher than those in Y09. Both objects are hard sources and, in particular, the one falling in the Chandra ACIS-I field appears to be absorbed by $N_{\mathrm{H}}>10^{22} \mathrm{~cm}^{-2}$. We speculate that the likely reason for the mismatch in the $2-10 \mathrm{keV}$ fluxes is that in Y09 the $\mathrm{X}$-ray fluxes of objects with only a few photons are obtained by using a photon index fixed to 1.9 , which, for hard sources may severely underestimate the X-ray flux above $2 \mathrm{keV}$. Using the correct $2-10 \mathrm{keV}$ fluxes, the two objects have $\mathrm{X} / \mathrm{NeV}$ values between 40 and 60 . The $\mathrm{X} / \mathrm{NeV}$ ratios of the 13 lowsignificance XMM-Newton detections and $\mathrm{X} / \mathrm{NeV}$ upper limits to the 3 XMM-Newton non-detections are shown in Fig. 2. They have been placed at $\log N_{\mathrm{H}}=21.5$, since for the majority of these objects there is evidence of absorption in their optical SDSS spectra. Their X/NeV values are distributed from $\sim 20$ to $\sim 300$; i.e., they are shifted towards lower values than those of local Seyfert 1s and of high-significance XMM-Newton detections in Y09. In principle, if broad line QSOs reach $\mathrm{X} / \mathrm{NeV}$ ratios as low as $\sim 20$, then one might suspect that more obscured sources, e.g. those absorbed by columns around $\sim 10^{23} \mathrm{~cm}^{-2}$ could easily contaminate the $\mathrm{CT}$ regime $(\mathrm{X} / \mathrm{NeV}<15)$ defined in the previous section. We note, however, that, as discussed above, the $\mathrm{X} / \mathrm{NeV}$ value could have been underestimated for many of the low-significance XMM-Newton detections in Y09. Furthermore, the two XMM-Newton non-detections with X/NeV 20 appear 
in the optical as pure narrow-line Seyferts and are in fact also listed in the Reyes et al. (2008) type-2 QSO catalog. Most likely, both objects are significantly absorbed, even CT, so we show them as lower limits on $N_{\mathrm{H}}$ in Fig. 2. Based on these checks, we conclude that the CT region defined in the previous section is not contaminated by SDSS blue, unabsorbed QSOs although some mild contamination by moderately absorbed sources might still be possible.

\subsection{SDSS type-2 QSOs at $z \sim 0.5$}

We then considered the objects presented by V10 (see also Vignali et al. 2006), who combined proprietary and archival X-ray observations of a sample of 25 objects out of 291 [O III]5007-selected type-2 QSOs (Zakamska et al. 2003). The objects in the V10 sample populate the redshift interval $z \sim[0.3-0.7]$ and have [O III] luminosities in excess of $1.9 \times$ $10^{9} L_{\odot}$. Based on the observed X/OIII ratio, V10 divided their sample into 8 Compton-thin QSOs and 17 CT QSOs candidates. We considered here the 21 objects in which the [Ne V] line is detected at $>3 \sigma$ level. Visual inspection of these $21 \mathrm{ob}-$ jects does not show any fake [Ne V] emission. We placed the V10 objects in the $\mathrm{X} / \mathrm{NeV}$ vs. $N_{\mathrm{H}}$ diagnostic diagram (see Fig. 2). An almost perfect correspondence is found between the $\mathrm{X} / \mathrm{OIII}$ and $\mathrm{X} / \mathrm{NeV}$ classifications, and all 8 objects classified as Compton-thin by V10 are also classified as Compton-thin based on their $\mathrm{X} / \mathrm{NeV}$ ratio. Conversely, 11 out of 13 objects that appear as CT candidates in V10 are also classified as CT using the $\mathrm{X} / \mathrm{NeV}<15$ threshold. This may not be surprising given that both [O III] and [Ne V] lines should trace the intrinsic AGN power. However, this is one proof that the $\mathrm{X} / \mathrm{NeV}$ ratio can be used as a diagnostic for revealing heavy obscuration. In Fig. 2 we also show the position of the distant $(z=0.44)$ CT QSO candidate IRAS 09104+4109 (Franceschini et al. 2000; Iwasawa et al. 2001a; Piconcelli et al. 2007b), whose SDSS optical spectrum reveals a clear [Ne V]3426 emission line. IRAS 09104 falls in a region of the $\mathrm{X} / \mathrm{NeV}$ diagram that, based on the local Seyfert sample presented in Sect. 3, is equally populated by CT and Compton-thin objects 5 .

The source distribution of SDSS objects in Fig. 2 appears to be less dispersed than that of local Seyferts in Fig. 1. This may be related to i) SDSS objects being intrinsically more luminous, hence less variable, than local Seyferts, and ii) being located at higher redshifts, which allows the SDSS $3^{\prime \prime}$ fibers to encompass the whole $[\mathrm{Ne} \mathrm{V}]$-emitting region, thus reducing aperture issues.

\section{SDSS obscured QSOs at $z \sim 1$}

\subsection{Sample selection}

To directly apply the $\mathrm{X} / \mathrm{NeV}$ obscuration diagnostic to distant AGN, we tried to select SDSS obscured QSOs at $z \sim 1$ on the basis of their [Ne V] emission. We searched in the SDSS DR7 entire spectroscopic database (table SpecPhotoAll on the SDSS site) for objects with high [Ne V] $E W$, since nuclear obscuration should suppress the AGN continuum but not the flux of the lines produced in the NLR.

We concentrated on objects with $0.8<z<1.4$, i.e., those not accessible through [O III] selection (thus excluded from the Zakamska et al. 2003; and Reyes et al. 2008 samples), and in

5 The CT nature of IRAS 09104 has been questioned by Piconcelli et al. (2007b), who nonetheless measured heavy absorption $\left(\log N_{\mathrm{H}} \sim\right.$ $\left.5 \times 10^{23} \mathrm{~cm}^{-2}\right)$ in this source.

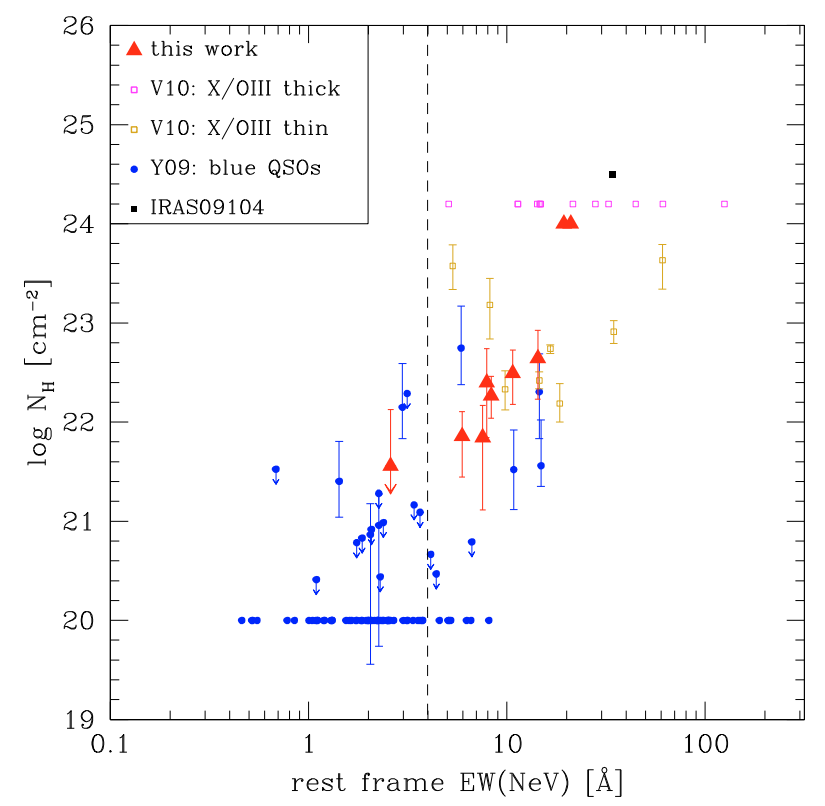

Fig. 3. Relation between rest frame equivalent width of the $[\mathrm{Ne} \mathrm{V}]$ line and X-ray obscuration for the various samples of SDSS QSOs considered in this work. Symbols are as in Fig. 2. The equivalent width of the $[\mathrm{Ne} \mathrm{V}$ ] line clearly correlates with X-ray obscuration. The dashed line shows the $E W_{\mathrm{NeV}}>4 \AA$ limit used to select the Chandra targets analyzed in this paper.

which the $[\mathrm{Ne} \mathrm{V}]$ line is observed at $\lambda<8200 \AA$, where the SDSS spectral efficiency is highest. To avoid spurious detections, we conservatively considered those objects in which the $[\mathrm{Ne} \mathrm{V}]$ line is detected at $\geq 5 \sigma$ in the SDSS spectral tables and, to avoid strong contamination from unobscured, broad line (type-1) AGN, we restricted the search to those spectra with MgII 2800 emission with $F W H M \lessgtr 3000 \mathrm{~km} \mathrm{~s}^{-1}$. The cut in the MgII 2800 line width is not stringent, and this leaves in the sample obscured QSO candidates with broad optical lines and a red continuum, such as the red-QSO population described e.g. by Wilkes et al. (2002, 2005). The resulting median, rest-frame [Ne V] $E W$ of the sample is $2 \AA$. We considered the high $E W$ tail of the distribution, i.e. the objects with $E W>4 \AA$ in the SDSS spectroscopic tables ( 89 objects). In Fig. 3 we show the correlation between the [Ne V] $E W$ and the X-ray absorption as measured for the SDSS QSO and type-2 QSO samples discussed in the previous sections: most objects with $E W>4 \AA$ do show significant X-ray absorption, validating the use of this $E W$ threshold $^{6}$.

We visually inspected all the 89 selected spectra to verify possible problems related to the SDSS automated procedure and to discard QSOs showing a blue continuum. At the end of this process, we selected 27 objects that appear to be good obscured QSO candidates at $z \sim 1$ based on their optical spectrum.

\subsection{Chandra observations}

In Chandra Cycle 10, we were awarded 10 ks ACIS-S observations of 8 targets that, based on their optical spectrum, were

\footnotetext{
${ }^{6}$ In Fig. 3 the 9 [Ne V]-selected targets discussed in the next section are also shown. After refitting the SDSS spectra, one object shows $E W \sim 2.6 \AA$, i.e., below the $E W>4 \AA$ threshold chosen in the SDSS spectroscopic tables. The re-fitted [Ne V] fluxes and $E W \mathrm{~s}$ of the remaining 8 objects are instead found to differ by only a few percent from those resulting from the SDSS automated fit procedure.
} 
Table 1. Basic sample properties.

\begin{tabular}{lcccr}
\hline \hline SDSS Name & $z$ & $R$ & $f_{\mathrm{NeV}}$ & $E W_{\mathrm{NeV}}$ \\
$(1)$ & $(2)$ & $(3)$ & $(4)$ & $(5)$ \\
\hline J105951.36+301817.4 & 0.887 & 19.667 & 8.12 & 8.3 \\
J092640.67+023628.7 & 1.306 & 20.658 & 7.76 & 14.3 \\
J034222.54-055727.9 & 0.882 & 19.846 & 6.83 & 7.5 \\
J104603.17+071907.2 & 0.888 & 20.191 & 5.73 & 10.7 \\
J165158.61+432508.6 & 0.853 & 20.093 & 4.20 & 5.9 \\
J080859.33+204711.8 & 0.908 & 21.059 & 4.98 & 21.0 \\
J125848.58+120531.1 & 1.035 & 20.013 & 5.13 & 7.9 \\
J145503.94+515539.9 & 1.277 & 19.466 & 2.00 & 2.6 \\
J085600.88+371345.5 & 1.022 & 21.278 & 4.05 & 19.3 \\
\hline
\end{tabular}

Notes. Column description: (1) Source name. (2) Redshift. (3) SDSS PSF magnitude in the $R$-band. (4) Measured [Ne V]3426 flux in units of $10^{-16} \mathrm{erg} \mathrm{cm}^{-2} \mathrm{~s}^{-1}$. (5) [Ne V]3426 rest frame equivalent width in units of Angstrom.

considered as good obscured QSOs targets (i.e., they show either narrow emission lines only or broad, $2000<F W H M<$ $3000 \mathrm{~km} \mathrm{~s}^{-1}$, lines over a red continuum). Observations were performed between December 2008 and July 2009 with the S3 chip at the aimpoint and using the very faint telemetry mode. We performed standard data reduction and extraction of spectra using CIAO v3.2.2. A $10 \mathrm{ks}$ ACIS-S observation of an archival object (SDSS J085600, PI Strauss) satisfying our selection criteria was also analyzed. The basic optical properties of these 9 objects (redshift, $R$-band magnitude, [Ne V] flux, and restframe $E W$ ) are shown in Table 1, while the journal of Chandra observations is shown in Table 2.

Apart from the archival object, which only shows 2 X-ray photons within $1.5^{\prime \prime}$ of the optical source position, all the other targets were detected, with photon statistics ranging from 18 to 313 counts in the $0.5-8 \mathrm{keV}$ band. Spectral fitting was performed using XSPEC v11.3.1. Standard $\chi^{2}$ minimization was adopted for spectra showing more than 100 counts in the 0.5-8 keV band, binned to a minimum of 10 counts per energy bin. C-statistics (Cash 1979) was used for spectra with lower photon statistics, binned to a minimum of 3 counts per bin. Model fits consist of simple power laws modified by Galactic absorption and absorption at the source redshift. Errors on the best-fit spectral parameters are quoted at $90 \%$ confidence level for one interesting parameter.

Four sources were detected with enough photon statistics (>100 counts) to perform a rough spectral analysis and classify them as Compton-thin, as also suggested by their low hardness ratios $(\mathrm{HR} \leq-0.3)$. One object, namely SDSS J080859, shows a faint (18 counts), very hard $(\mathrm{HR}=0.8)$ spectrum with a hint of excess emission at $\sim 3.3 \mathrm{keV}$. If the excess is interpreted as a redshifted $6.4 \mathrm{keV}$ iron $\mathrm{K} \alpha$ emission line superimposed to a hard power-law continuum $(\Gamma=-1.2)$, this would imply a rest frame equivalent width of $E W=2.8_{-2.1}^{+3.3} \mathrm{keV}$. The X-ray spectral features of SDSS J080859 are therefore highly suggestive of CT absorption. Three objects (SDSS J092640, SDSS J104603, and SDSS J125848) are intermediate cases in terms of both photon statistics (from 18 to 65 counts) and $\mathrm{X}$-ray hardness (from $\mathrm{HR}=-0.16$ to -0.05 ). By fitting the data with an absorbed power law (fixing $\Gamma$ to 1.8 for two of them), these sources appear to be Compton-thin. All the detected sources have relatively bright observed $2-10 \mathrm{keV}$ fluxes $\left(>3 \times 10^{-14} \mathrm{erg} \mathrm{cm}^{-2} \mathrm{~s}^{-1}\right)$ and rest-frame $2-10 \mathrm{keV}$ luminosities $\gtrsim 10^{44} \mathrm{erg} \mathrm{s}^{-1}$ (prior to absorption corrections), placing them among luminous obscured QSOs. As for the archival undetected
Table 2. Log of X-ray observations.

\begin{tabular}{lrrc}
\hline \hline $\begin{array}{l}\text { SDSS Name } \\
(1)\end{array}$ & $\begin{array}{r}\text { ObsId } \\
(2)\end{array}$ & $\begin{array}{r}\text { Date } \\
(3)\end{array}$ & $\begin{array}{c}\text { Exp. } \\
(4)\end{array}$ \\
\hline J105951.36+301817.4 & 10325 & $2009-01-29$ & 10.0 \\
J092640.67+023628.7 & 10326 & $2009-03-23$ & 10.0 \\
J034222.54-055727.9 & 10327 & $2008-12-02$ & 9.8 \\
J104603.17+071907.2 & 10328 & $2009-02-10$ & 10.0 \\
J165158.61+432508.6 & 10329 & $2009-06-30$ & 10.0 \\
J080859.33+204711.8 & 10330 & $2008-12-22$ & 10.0 \\
J125848.58+120531.1 & 10331 & $2009-02-23$ & 9.2 \\
J145503.94+515539.9 & 10332 & $2009-07-27$ & 10.0 \\
J085600.88+371345.5 & 6807 & $2006-02-17$ & 10.3 \\
\hline
\end{tabular}

Notes. Column description: (1) Source name. (2) Chandra observation identifier. (3) Observing date (yyyy-mm-dd). (4) Exposure time in ks.

object, SDSS J085600, by assuming a 2-10 keV band detection limit of 4 photons and a pure reflection spectrum (pexrav in XSPEC), the corresponding upper limits to the $2-10 \mathrm{keV}$ flux and rest frame luminosity, are $2 \times 10^{-14} \mathrm{erg} \mathrm{cm}^{-2} \mathrm{~s}^{-1}$ and $3 \times$ $10^{43} \mathrm{erg} \mathrm{s}^{-1}$, respectively. A summary of the results obtained from the X-ray analysis of these 9 objects is given in Table 3. Optical and X-ray spectra are shown in Fig. 4

\subsection{Position on the $\mathrm{X} / \mathrm{NeV}$ diagram}

We placed the new 9 SDSS obscured QSOs at $z \sim 1$ on the $\mathrm{X} / \mathrm{NeV}$ diagram (see Fig. 2). The archival undetected X-ray object SDSS J085600 shows $\mathrm{X} / \mathrm{NeV}<15$, which is strongly suggestive of CT absorption. Indeed, most SDSS QSOs classified as CT by V10 do show the X/NeV ratio below this value, while all those classified as Compton-thin by V10 lie at X/NeV > 100 .

The faint (18 X-ray counts) object SDSS J080859 has $\mathrm{X} / \mathrm{NeV}=45$. This value was observed in both $\mathrm{CT}$ and Comptonthin AGN (see Fig. 1), but based on the X-ray spectral analysis, SDSS J080859 appears to have all the signatures of CT absorption. We then classify SDSS J085600 and SDSS J080859 as likely CT candidates.

The four objects with highest photon statistics also have the lowest absorption and the highest $\mathrm{X} / \mathrm{NeV}$ ratios $(\mathrm{X} / \mathrm{NeV}>150)$, and occupy a region of the diagram that is populated by the Compton-thin QSOs in V10 and by the obscured tail of the broad line SDSS QSOs studied by Young et al. (2009a).

Finally, three objects do show $\mathrm{X} / \mathrm{NeV}$ ratios in the range 20-60, which are typical of heavily obscured AGN, but are still consistent with Compton-thin absorption. Both the X-ray and optical spectra of these sources would favor mild absorption, hence a Compton-thin interpretation, although no clear cut classification can be made with the current data.

The actual degree of obscuration of our two CT candidates SDSS J085600 and SDSS J080859 can only be confirmed by obtaining good-quality X-ray spectra through deeper observations. Indeed, any selection method that simply relies on X-ray hardness ratios or on the comparison between the measured, obscured X-ray emission (if any) with some other indicator of the intrinsic nuclear power (e.g. dust-reprocessed IR-emission, or high-ionization, narrow optical emission lines) can only provide an indirect way to select CT AGN. Different caveats and limitations affect these different selection methods, such as reddening in line-selected sources and contamination from star formation in IR-selected sources. 
Table 3. Results from X-ray data analysis and spectral fitting.

\begin{tabular}{|c|c|c|c|c|c|c|c|c|c|c|}
\hline $\begin{array}{l}\text { SDSS Name } \\
\text { (1) }\end{array}$ & $\begin{array}{r}\text { Cts } \\
(2)\end{array}$ & $\begin{array}{l}\mathrm{HR} \\
(3)\end{array}$ & $\begin{array}{c}N_{\mathrm{H}}^{\mathrm{gal}} \\
(4)\end{array}$ & $\begin{array}{l}N_{\mathrm{H}} \\
(5)\end{array}$ & $\begin{array}{c}\Gamma \\
(6)\end{array}$ & $\begin{array}{r}\chi^{2} / \text { d.o.f. } \\
\text { (7) }\end{array}$ & $\begin{array}{r}f_{2-10} \\
(8)\end{array}$ & $\begin{array}{l}L_{2-10}^{\mathrm{abs}} \\
(9)\end{array}$ & $\begin{array}{l}L_{2-10}^{\text {int }} \\
(10)\end{array}$ & $\begin{array}{l}\text { Class } \\
\text { (11) }\end{array}$ \\
\hline $\mathrm{J} 105951.36+301817.4$ & 237 & -0.30 & 2.0 & $1.84_{-0.75}^{+1.05}$ & $1.90_{-0.35}^{+0.40}$ & $25.6 / 20$ & 16 & 44.70 & 44.80 & thin \\
\hline J092640.67+023628.7 & 65 & -0.06 & 3.8 & $4.40_{-2.70}^{+4.00}$ & $1.67_{-0.65}^{+0.75}$ & $\ldots$ & 6.8 & 44.61 & 44.75 & thin? \\
\hline J034222.54-055727.9 & 175 & -0.50 & 5.3 & $0.70_{-0.57}^{+0.77}$ & $1.83_{-0.45}^{+0.05}$ & $10.4 / 13$ & 13 & 44.60 & 44.61 & thin \\
\hline $\mathrm{J} 104603.17+071907.2$ & 31 & -0.16 & 2.8 & $3.10_{-1.59}^{+2.22}$ & $1.8^{\text {fixed }}$ & $\cdots$ & 3.7 & 44.04 & 44.15 & thin? \\
\hline $\mathrm{J} 165158.61+432508.6$ & 313 & -0.38 & 1.9 & $0.72_{-0.44}^{+0.55}$ & $1.76_{-0.30}^{+0.33}$ & $20.7 / 27$ & 19 & 44.76 & 44.79 & thin \\
\hline J080859.33+204711.8 & 18 & 0.80 & 4.0 & $\ldots$ & $-1.2_{-1.4}^{+0.90}$ & $\ldots$ & 14 & 43.95 & $45.65^{*}$ & thick \\
\hline $\mathrm{J} 125848.58+120531.1$ & 18 & -0.05 & 2.2 & $2.5_{-1.8}^{+3.0}$ & $1.8^{\text {fixed }}$ & $\ldots$ & 1.8 & 43.88 & 43.97 & thin? \\
\hline $\mathrm{J} 145503.94+515539.9$ & 125 & -0.43 & 1.8 & $0.36_{-0.96}^{+0.08}$ & $1.82_{-0.41}^{+0.58}$ & $14.4 / 9$ & 5.7 & 44.67 & 44.69 & thin \\
\hline J085600.88+371345.5 & $<4$ & $\ldots .$. & 2.9 & $\ldots$ & $\ldots$ & $\ldots$ & $<2.0$ & $<43.50$ & $<45.20^{*}$ & thick \\
\hline
\end{tabular}

Notes. Column description: (1) Source name. (2) Net counts in the $0.5-8 \mathrm{keV}$ band. (3) Hardness ratio, defined as $(\mathrm{H}-\mathrm{S}) /(\mathrm{H}+\mathrm{S})$, where $\mathrm{H}$ and $\mathrm{S}$ are the number of photons observed in the $0.5-2 \mathrm{keV}$ and $2-8 \mathrm{keV}$ bands, respectively. (4) Galactic column density in units of $10^{20} \mathrm{~cm}^{-2}$ (Dickey \& Lockman 1990). (5) Absorbing column density at the source redshift in units of $10^{22} \mathrm{~cm}^{-2}$. (6) Photon index. (7) Chi-square value over number of degrees of freedom: for sources with less than 100 net counts C-statistics has been used. (8) Observed 2-10 keV flux in units of $10^{-14} \mathrm{erg} \mathrm{cm}^{-2} \mathrm{~s}^{-1}$. (9) Logarithm of the $2-10 \mathrm{keV}$ rest frame luminosity, not corrected for absorption, in units of erg s $\mathrm{s}^{-1}$. (10) Logarithm of the intrinsic 2-10 keV rest frame luminosity, i.e. corrected for absorption, in units of $\mathrm{erg} \mathrm{s}^{-1}$; * for the two candidate Compton-thick objects it is assumed $L^{\text {int }}=50 \times L^{\text {obs }}$ (see text). (11) Object classification based on the X-ray spectral analysis and the $\mathrm{X} / \mathrm{NeV}$ ratio.

\section{Discussion}

\section{1. [Ne V] as a good tracer of nuclear luminosity: effects of reddening in the narrow line region and anisotropy}

The $\mathrm{X} / \mathrm{NeV}$ vs. X-ray absorption diagnostic diagram presented in Sect. 3 was derived directly from observed values, without applying any corrections to either $[\mathrm{Ne} \mathrm{V}]$ or X-ray emission. The rationale behind it is that $\mathrm{X}$-rays come from the innermost nuclear regions and can be depressed by small-scale $(<1 \mathrm{pc})$ absorption, while the $[\mathrm{Ne} \mathrm{V}]$ is instead a good indicator of the intrinsic nuclear luminosity being i) emitted on larger (kpc) scales, free of nuclear obscuration and ii) isotropic. We now discuss whether these two hypotheses are satisfied and what happens if they are not.

As for the first point, we note that significant extinction towards the NLR is commonly observed in local AGN (e.g. Dahari $\&$ De Robertis 1988). In the simplest version of the AGN unification schemes, one would expect to observe similar properties in the NLR of both type-1 and type-2 AGN. Therefore, if the dust content in the NLR of both AGN types is the same, the same extinction correction should be applied to the measured values of the [Ne V] lines, which would simply shift all the datapoints in Figs. 1 and 2 towards lower $\mathrm{X} / \mathrm{NeV}$ values, without altering the $\mathrm{X} / \mathrm{NeV}$ vs. $N_{\mathrm{H}}$ trend. However, based on the results by Dahari \& De Robertis (1988), there is some evidence that the extinction to the NLR in local Seyfert $2 \mathrm{~s}$ is somewhat higher than in Seyfert 1s, the following relation holding for the median values: $A_{V}^{\text {Sy2 }}-A_{V}^{\text {Sy1 }} \sim 1.5-1.0=0.5$. Using standard extinction curves (e.g. Gaskell \& Benker 2007), this translates into an average correction in the $[\mathrm{Ne} \mathrm{V}]$ flux a factor of 2.3 larger in Seyfert 2s than in Seyfert 1s. Applying some extinction correction to both the observed data and model curves would then produce a stronger shift towards lower $\mathrm{X} / \mathrm{NeV}$ ratios in Seyfert 2s than in Seyfert 1s, making the anti-correlation between $\mathrm{X} / \mathrm{NeV}$ ratio and obscuration even more evident.

At any rate, our main interest is in the possibility of applying the $\mathrm{X} / \mathrm{NeV}$ diagnostic ratio to $z \sim 1$ objects, for which it is often impossible to measure the extinction to the NLR because of the lack of strong Balmer lines in the optical spectrum. Therefore, we simply do not apply any reddening correction to the measured [Ne V] fluxes.

As for the second point, the isotropy of [ $\mathrm{Ne} \mathrm{V}]$ emission, there is some evidence that the [Ne V] lines in SDSS type-1 QSOs are broader than in type-2 QSOs. As an example, in the composite spectrum of QSOs from the $2 \mathrm{dF}$ survey (Colless et al. $2001)$, the $[\mathrm{Ne} \mathrm{V}]$ width $\left(F W H M \sim 1200 \mathrm{~km} \mathrm{~s}^{-1}\right)$ is found to be in-between that of broad lines (e.g. $\mathrm{H} \beta$ and $\mathrm{MgII}, F W H M \sim$ $4000 \mathrm{~km} \mathrm{~s}^{-1}$ ) and that of narrow lines (e.g. [O II] and[O III], $F W H M \sim 600 \mathrm{~km} \mathrm{~s}^{-1}$; see Fig. 2 in Corbett et al. 2003).

According to the standard unified model, this would suggest that, whenever a direct look towards the nucleus is available, an additional broader [ $\mathrm{Ne} \mathrm{V}$ ] component is observed, coming from low-density and fast-moving clouds that cannot be observed in type-2 AGN, possibly because they are located inbetween the BLR and the NLR. If this is true, then the total $[\mathrm{Ne} \mathrm{V}]$ emission line is not a good isotropic indicator of the intrinsic AGN power, since only a portion of the [Ne V] emitting region can be observed in type- 2 objects. If anything, this would go in the direction of underestimating the total [ $\mathrm{Ne} \mathrm{V}]$ luminosity in type-2 AGN. Correcting for this effect would make the $\mathrm{X} / \mathrm{NeV}$ ratio even lower in type- $2 \mathrm{AGN}$. To summarize, if corrections for reddening and anisotropy were applied, the $\mathrm{X} / \mathrm{NeV}$ vs. $N_{\mathrm{H}}$ trend shown in Fig. 2 would be even stronger.

\subsection{Comparison between [O III] and [Ne V] selection}

As discussed in Sect. 4.2, 2 out of 9 [Ne V]-selected type-2 QSOs $(\approx 22 \%)$ appear to be likely CT candidates based on their $\mathrm{X}$-ray spectral properties and/or $\mathrm{X} / \mathrm{NeV}$ ratio. At face value, the fraction of $[\mathrm{Ne} \mathrm{V}]$-selected CT QSO candidates appears lower than when based on [O III] selection, which is around $60-70 \%$ (V10). However, we note that the difference between the CT detection rate can be ascribed mainly to intrinsic differences in the two parent samples of obscured QSOs. Indeed, our sample also includes obscured QSOs that have a red continuum but still show broad optical lines. These objects (see Fig. 4) 
R. Gilli et al.: [Ne V] selected obscured QSOs
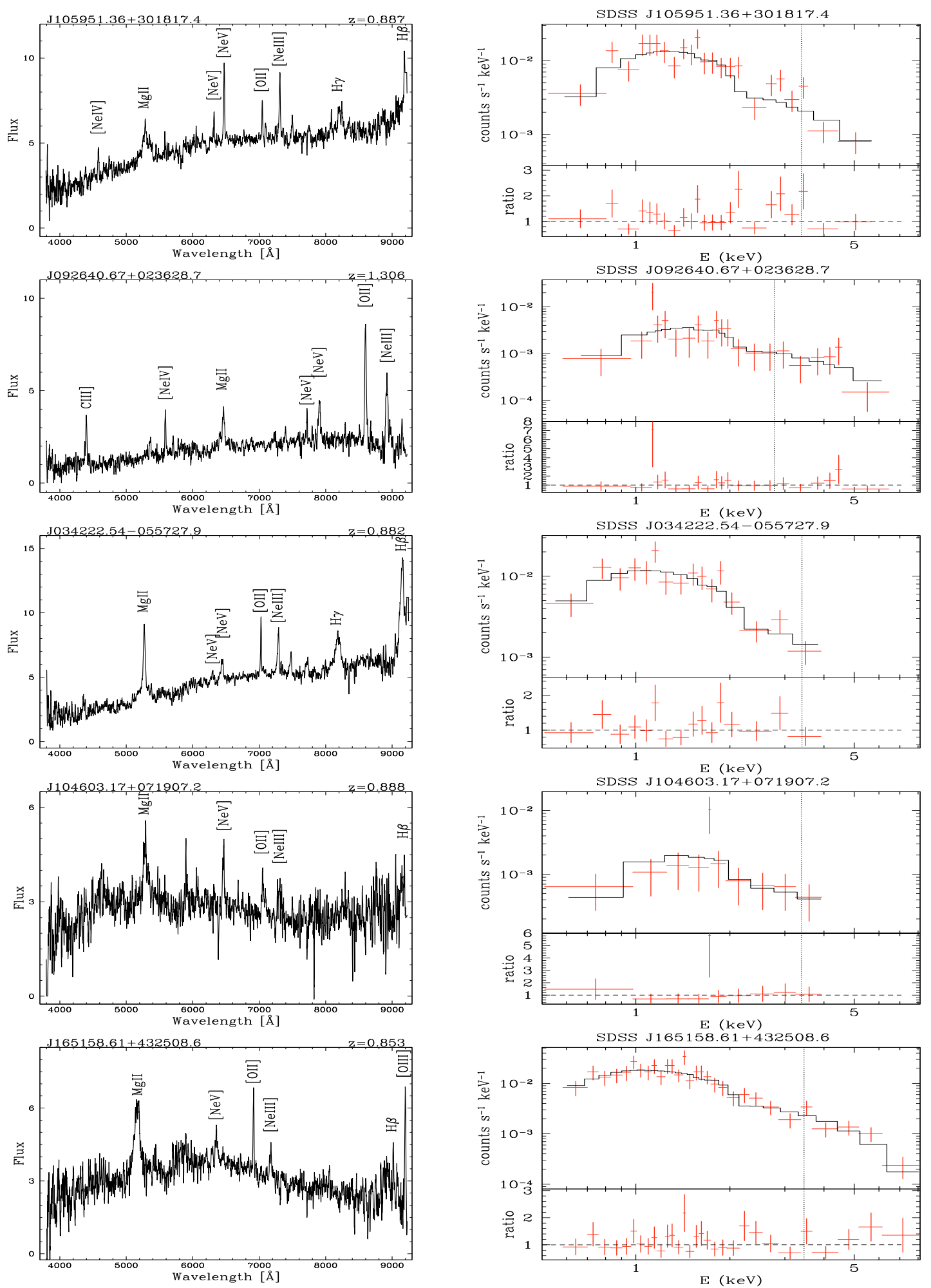

Fig. 4. The SDSS optical spectra (left) and Chandra X-ray spectra (right) of the 9 obscured QSOs at $z \sim 1$ presented in this work. Both optical and X-ray spectra are plotted in the observed frame. Flux units for the SDSS spectra are $10^{-17} \mathrm{erg} \mathrm{cm}^{-2} \mathrm{~s}^{-1} \AA^{-1}$. The dotted vertical lines in the right panels mark the expected position in the observed frame of the $6.4 \mathrm{keV} \mathrm{Fe} \mathrm{K \alpha}$ line. The archival object SDSS J085600 is not detected in the X-rays: we show a $30^{\prime \prime} \times 30^{\prime \prime}$ Chandra image in the $0.5-8 \mathrm{keV}$ band around the source position (marked with a $1.5^{\prime \prime}$ radius circle).

appear to be part of the population of red QSOs discussed e.g. by Wilkes et al. $(2002,2005)$ and Urrutia et al. (2005), which on average are absorbed by columns below $N_{\mathrm{H}}=10^{23} \mathrm{~cm}^{-2}$. On the contrary, V10 selected their objects from the sample of
Zakamska et al. (2003), in which strict criteria on the line width have been adopted, producing an ensemble of pure type- $2 \mathrm{ob}-$ jects. As a simple check, when considering only those [Ne V]selected objects that look like pure type-2 spectra (e.g. MgII 

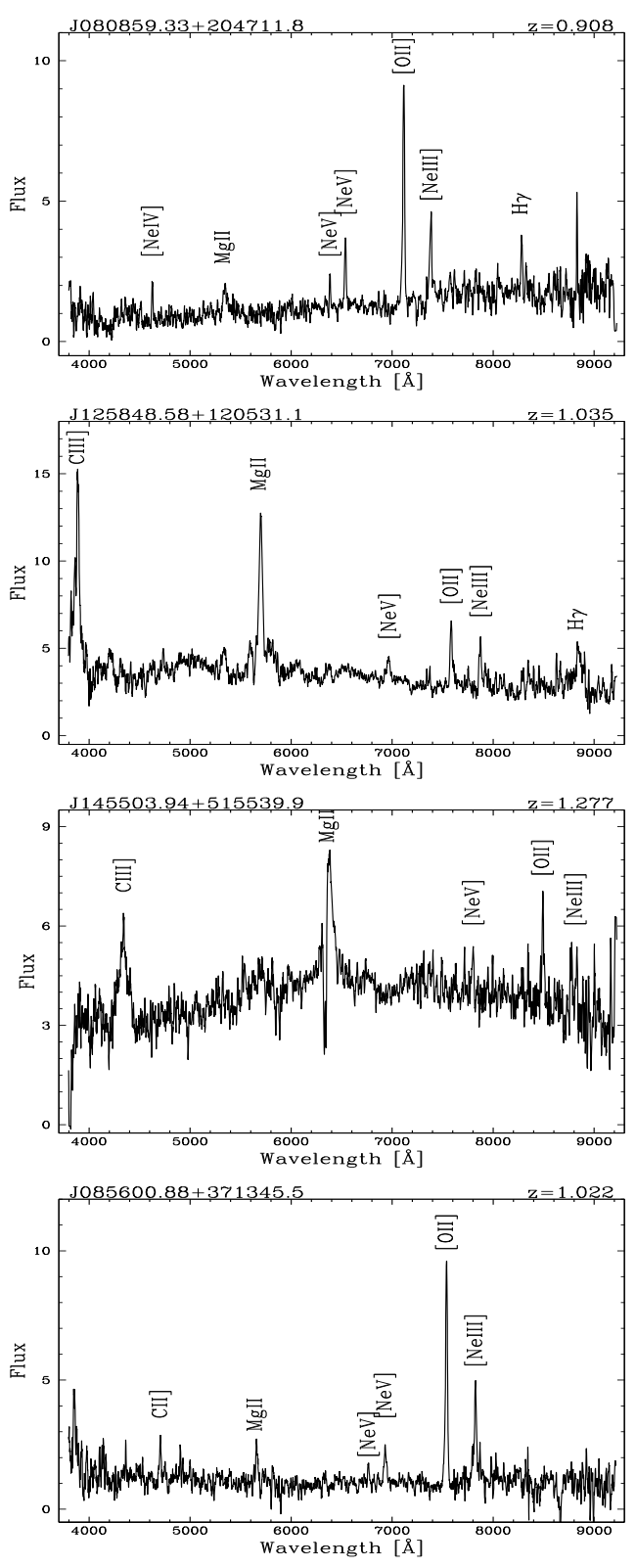

Fig. 4. continued.

$F W H M \lesssim 2000 \mathrm{~km} \mathrm{~s}^{-1}$ ), the fraction of CT candidates increases to $\approx 50 \%(2 / 4)$. Despite the very low statistics, this fraction is consistent with what has been found by V10, suggesting that, when the search is restricted to pure type-2 objects, [Ne V] selection may be an efficient way to pick up CT AGN at $z \sim 1$.

\subsection{Application of the $\mathrm{X} / \mathrm{NeV}$ diagnostic to spectroscopic surveys with deep $X$-ray coverage}

Synthesis models predict that from $10 \%$ to about $30 \%$ of the $\mathrm{XRB}$ at $30 \mathrm{keV}$ is not accounted for by the integrated emission of Compton-thin AGN. This "missing" background is expected to be produced by CT AGN, and most of it is expected to be produced by CT AGN with Seyfert-like intrinsic luminosities at a redshift of $z \sim 1$. The [Ne V]-selected CT QSOs in the SDSS represent the high-luminosity, low space density tail of the distribution of CT AGN at $z \sim 1$, and are expected to
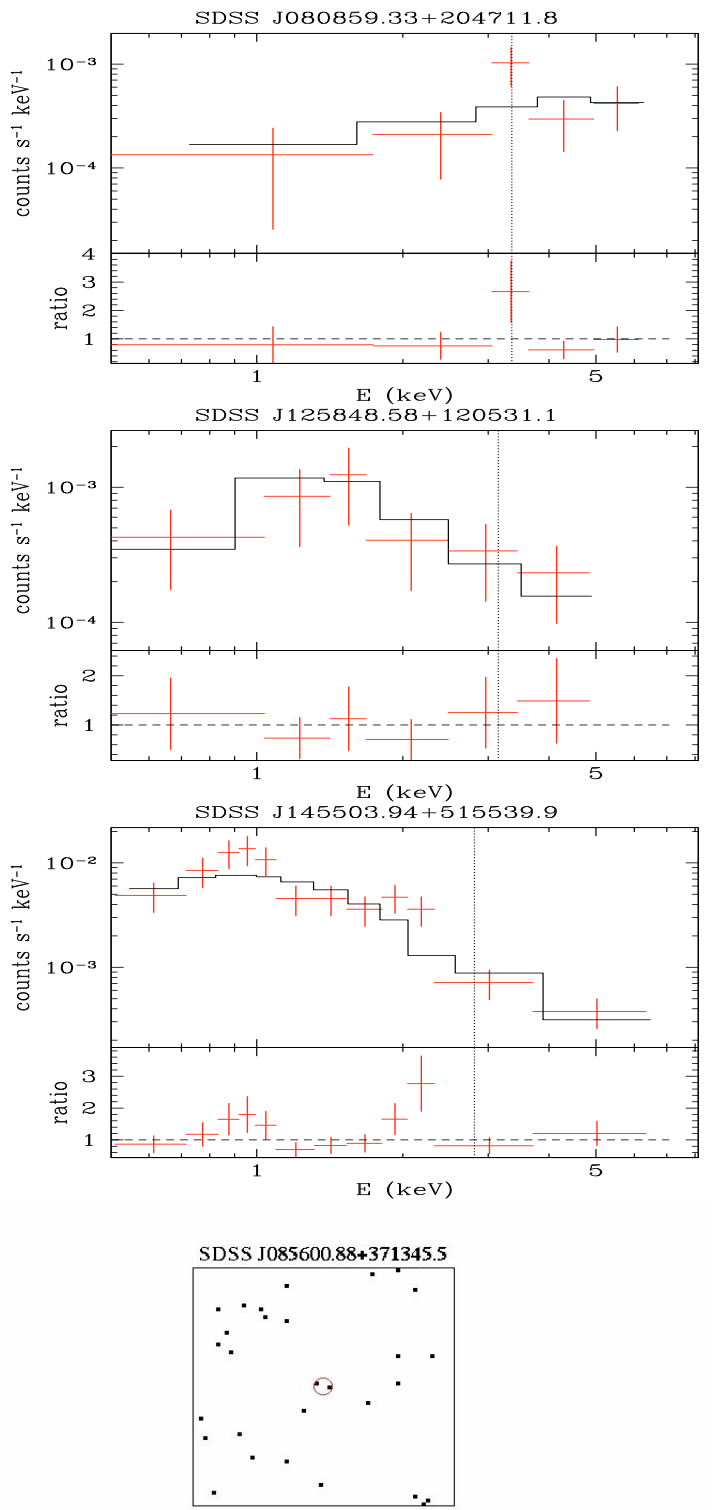

make only a minor contribution to the missing XRB. Selection of lower-luminosity CT AGN at $z \sim 1$ is therefore needed, which can in principle be done by applying the $\mathrm{X} / \mathrm{NeV}$ diagnostics to objects in sky areas with deep spectroscopic surveys and deep X-ray coverage. As an example, the combination between the zCOSMOS-bright spectroscopic survey (Lilly et al. 2007, 2009) and the Chandra-COSMOS X-ray survey (Elvis et al. 2009; Puccetti et al. 2009) in the COSMOS field (Scoville et al. 2007) would be able to identify CT AGN at $z \sim 1$ down to intrinsic $L_{\mathrm{X}} \sim 10^{43} \mathrm{erg} \mathrm{s}^{-1}$, i.e., the population which is thought to produce a large fraction of the missing XRB (Vignali et al., in prep.). In the GOODS-S and -N fields, the population of CT AGN at $z \sim 1$ can be tracked farther down to intrinsic $L_{X}$ of $10^{42} \mathrm{erg} \mathrm{s}^{-1}$. Furthermore, since the CT samples obtained with the $\mathrm{X} / \mathrm{NeV}$ diagnostic appear to be relatively free from contamination by less obscured objects, by using spectroscopic surveys with well-defined selection functions it would be possible to estimate the space density of this missing AGN population. 
It has to be noted that selection of obscured AGN through the [Ne V]3426 line is most likely a lower limit, since it misses objects with dusty narrow line regions, which can instead be picked up by mid-IR selection (Daddi et al. 2007; Fiore et al. 2008). However, when sufficiently deep mid-IR coverage is not available for spectroscopic survey fields, [Ne V]-selection may then represent a promising and ready-to-use method to get large samples of $z \sim 1 \mathrm{CT}$ AGN. In addition, comparing the space density of [Ne V] and mid-IR selected objects with similar redshifts and bolometric luminosities in fields with full multiwavelength coverage may indicate the fraction of objects in which the NLR is free of obscuration, thus constraining the physical scale on which absorption arises.

\subsection{Enhanced star formation in type-2 QSOs?}

Popular semi-analytic models of galaxy formation and evolution (Kauffmann \& Haehnelt 2000; Marulli et al. 2008; Hopkins et al. 2008) propose that, at least for the most massive and luminous objects, nuclear activity and star formation are both triggered by major mergers of gas-rich galaxies, and that at the early stages of the merger, when star formation is more vigorous, the AGN is embedded within optically thick gas shrouds. According to this scenario one can therefore expect to observe strong star formation in obscured QSOs.

At redshift $z \sim 2$, the concurrent, obscured black hole growth and star formation have been observed in the population of bright submillimeter sources (e.g. Alexander et al. 2005). At low redshifts $(z<0.3)$, the ratio between the luminosity of the [O II]3727 line and the [O III]5007 line is a factor of $\sim 4$ higher in the type-2 QSO composite spectrum of Zakamska et al. (2003) than in the average spectrum of type-1 QSOs (Kim et al. 2006). Because of the similar [O III] luminosity of the two populations, the excess of low-ionized oxygen in type-2 QSOs could not be explained in terms of different ionization parameters of the narrow line regions, and was then interpreted by Kim et al. (2006) as the result of enhanced star formation in type-2 QSOs.

We then investigated the star formation in the samples presented in this work by measuring the [O II]3727 flux on the SDSS spectra and considered the [O II] over [Ne V] ratio as a function of the nuclear obscuration. To compare objects in the same redshift range, we restricted our analysis to $z>0.4$. As shown in Fig. 5, this cut removes only low-redshift type-1 QSOs and ensures that both type-1 and type-2 QSOs span a similar luminosity range ${ }^{7}$. For QSOs in the redshift range $z=$ $0.4-1.5$, the $3^{\prime \prime}$ size of the SDSS fibers encloses regions as large as $16-26 \mathrm{kpc}$ diameter, and therefore samples a significant portion of the host galaxy in which star formation can take place.

In Fig. 6 we plot the $[\mathrm{O} \mathrm{II}] /[\mathrm{Ne} \mathrm{V}]$ ratio as a function of the measured X-ray column density. The blue SDSS QSOs in the Y09 sample do show $[\mathrm{O} \mathrm{II]/[Ne} \mathrm{V]} \mathrm{ratios} \mathrm{that} \mathrm{are} \mathrm{on} \mathrm{average}$ lower than type-2 QSOs. Some positive correlation, albeit with a large scatter, is indeed seen between the $[\mathrm{O} \mathrm{II}] /[\mathrm{Ne} \mathrm{V}]$ ratio and the absorbing column density $N_{\mathrm{H}}$. When considering those objects with observed $[\mathrm{O} \mathrm{II}] /[\mathrm{Ne} \mathrm{V}]>4$, we found that only 2 out of 12 are not obscured, and half of them ( 6 objects) are likely to

\footnotetext{
7 The intrinsic X-ray luminosity of CT QSOs has been assumed to be $50 \times$ the observed one, while V10 defined as CT candidates those objectes in which the expected intrinsic X-ray luminosity was at least a factor of 100 more than the observed one. Even with a factor of 2 larger correction, the intrinsic luminosity of the CT QSOs considered in this work would still be comparable to that of unobscured QSOs in the same redshift range.
}

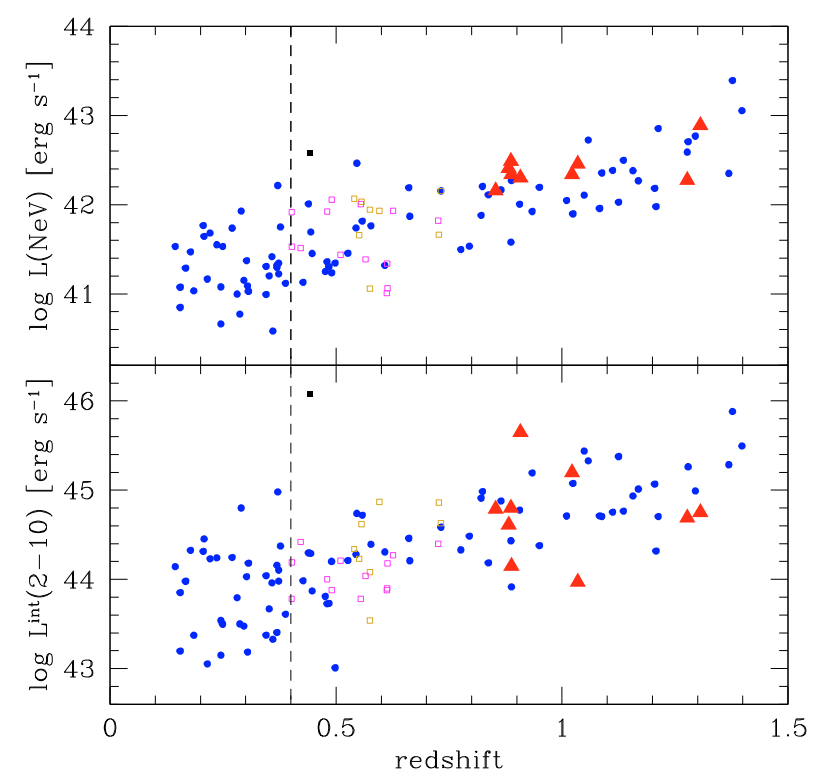

Fig. 5. [Ne V] luminosity (upper panel) and intrinsic 2-10 keV rest frame luminosity (lower panel) vs. redshift for the different SDSS QSO samples presented in this work. Symbols are as in the previous figures. For CT QSO candidates, we assumed that the intrinsic $2-10 \mathrm{keV}$ rest frame luminosity is a factor of 50 larger than the observed one. The dashed line shows the $z>0.4$ threshold adopted to compare the [O II], $[\mathrm{Ne} \mathrm{V}]$ and X-ray properties of QSOs of similar intrinsic luminosity (see text).

be obscured by CT absorption. Conversely, there are no objects with $N_{\mathrm{H}}>10^{23} \mathrm{~cm}^{-2}$ among those with [O II] $/[\mathrm{Ne} \mathrm{V}]<1$. The $[\mathrm{O} \mathrm{II}] /[\mathrm{Ne} \mathrm{V}]$ ratios measured on the SDSS type-2 and type-1 QSO composites by Zakamska et al. (2003) and Vanden Berk et al. (2001) were also considered and found to be in good agreement with the averages measured in this work for obscured and unobscured QSOs, respectively (see Fig. 6). We tried to compute the significance of the correlation between the $[\mathrm{O} \mathrm{II}] /[\mathrm{Ne} \mathrm{V}]$ ratio and the logarithm of the column density. It is difficult to deal with objects that are either unobscured or CT candidates, because they cannot be treated statistically as proper upper or lower limits on $N_{\mathrm{H}}$, since the gas column density can plausibly vary only within a bounded range (i.e. it cannot be zero or infinite). For simplicity we therefore assumed $\log N_{\mathrm{H}}=20$ for unobscured objects and $\log N_{\mathrm{H}}=24$ for CT candidates, respectively (see Fig. 6). A correlation has been estimated through the ASURV software package (Lavalley et al. 1992), using the generalized Kendall's $\tau$ and the Spearman's $\rho$ correlation tests. We found that the probability that the correlation is not present is only $2 \times 10^{-4}$ and $1 \times 10^{-4}$, respectively. If the [O II] emission measured in type-2 QSOs is interpreted as entirely due to star formation, the median [O II] luminosities of the [O III]- and [Ne V]-selected samples would correspond to star formation rates of $\approx 100$ and $\approx 200 M_{\odot} / \mathrm{yr}$, respectively (using the relation by Kewley et al. 2004). These values could decrease by up to a factor of $\sim 2$ if the AGN contribution to the [OII] emission is significant (Silverman et al. 2009). This finding is consistent with the expectations from the AGN evolutionary sequence outlined above.

\section{Conclusions}

We have presented a diagnostic diagram to identify heavily obscured, Compton-thick (CT) AGN candidates at $z \sim 1$ based on 


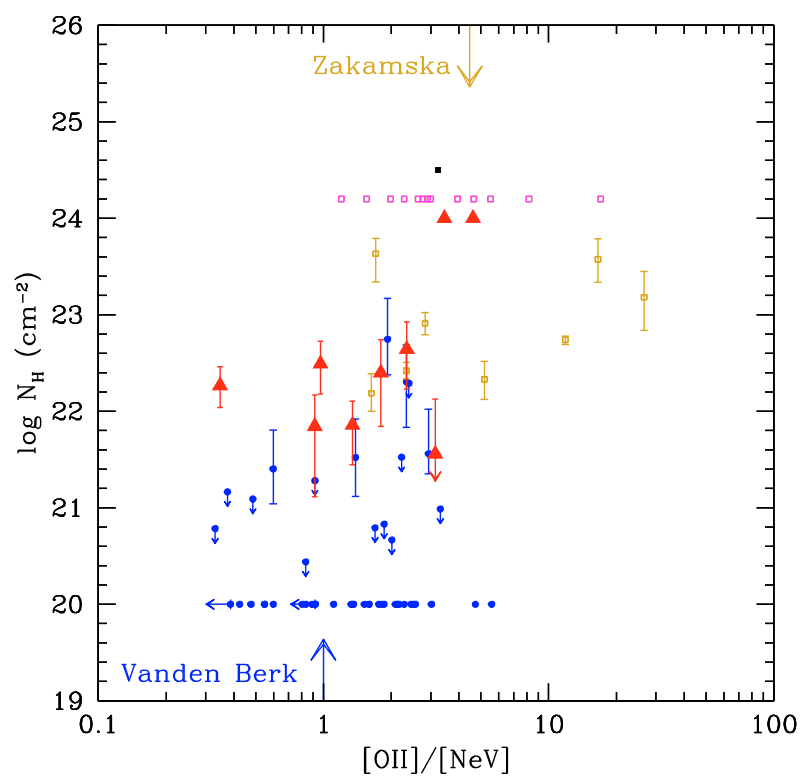

Fig. 6. [O II]/[Ne V] luminosity ratio vs. X-ray column density for SDSS QSOs and type-2 QSOs at $z>0.4$. Symbols are as in the previous figures. A trend is visible in which progressively more obscured objects have enhanced [O II] emission relative to [Ne V]. We interpret this trend as due to increased star formation in obscured QSOs, which boosts the $[\mathrm{O} \mathrm{II}]$ line emission. The $[\mathrm{O} \mathrm{II}] /[\mathrm{Ne} \mathrm{V}]$ ratio as measured on the SDSS type-1 QSO composite spectrum of Vanden Berk et al. (2001) and on the SDSS type-2 QSO composite spectrum of Zakamska et al. (2003) are shown with the two labeled arrows.

the ratio between the $2-10 \mathrm{keV}$ flux and the [Ne V]3426 emission line flux $(\mathrm{X} / \mathrm{NeV})$. The diagnostic was calibrated on a sample of 74 local Seyfert galaxies and then applied to populations of type-1 and type-2 QSOs at different redshifts (from $z \sim 0.1$ to $z=1.5$ ) selected from the SDSS. The main results obtained in this work can be summarized as follows.

- The observed $\mathrm{X} / \mathrm{NeV}$ ratio is found to decrease with increasing absorption: the mean $\mathrm{X} / \mathrm{NeV}$ ratio for unobscured Seyferts is about 400 , about $80 \%$ of local Seyferts with $\mathrm{X} / \mathrm{NeV}<100$ are obscured by column densities above $10^{23} \mathrm{~cm}^{-2}$ and essentially all objects with observed $\mathrm{X} / \mathrm{NeV}<15$ are CT.

- We considered a sample of 83 blue type-1 QSOs and 21 [O III]-selected type-2 QSOs in the SDSS that have been observed in the X-rays and show significant [Ne V] detection. It was verified that they follow the same $\mathrm{X} / \mathrm{NeV}$ vs. X-ray absorption trend observed for local Seyferts. Furthermore, SDSS type-2 QSOs classified either as CT or Compton-thin on the basis of their X/OIII ratio, would have mostly been classified in the same way based on the $\mathrm{X} / \mathrm{NeV}$ ratio.

- The $\mathrm{X} / \mathrm{NeV}$ diagnostic was used to investigate the obscuration of 9 SDSS obscured QSOs in the redshift range $z=$ [0.85-1.31], which is not accessible through [O III] selection. The 9 objects were selected by means of their prominent [Ne V]3426 line $(E W>4 \AA)$, and Chandra snapshot observations for 8 of them were obtained (one object is from the archive). Based on the $\mathrm{X} / \mathrm{NeV}$ ratio, complemented by $\mathrm{X}$-ray spectral analysis, only 2 objects appear good CT QSO candidates. However, when considering the 4 genuine narrowline objects only ( $F W H M$ of the MgII line $\$ 2000 \mathrm{~km} \mathrm{~s}^{-1}$ ), the efficiency in selecting CT QSOs through the [Ne V] line is about $50 \%(2 / 4)$, which is more similar, despite the large uncertainties, to what is achieved with [O III] selection (60-70\%; Vignali et al. 2010).

- We verified that neither extinction nor anisotropy corrections on the $[\mathrm{Ne} \mathrm{V}]$ emission would affect our conclusions and that the $\mathrm{X} / \mathrm{NeV}$ diagnostic is therefore a good method for identifying clean, even if not complete, samples of heavily obscured AGN. We discussed the possibility of applying the $\mathrm{X} / \mathrm{NeV}$ diagnostic to objects in sky areas with deep optical spectroscopy and X-ray coverage. This will allow CT Seyferts to be identified at $z \sim 1$, i.e., those objects thought to be responsible for a large fraction of the "missing" X-ray background.

- Finally, the optical emission line properties of [Ne V]-selected QSOs were compared with those of other SDSS populations of obscured and unobscured QSOs. By restricting the analysis to objects in the same redshift (and luminosity) range $z=[0.4-1.5]$, we found evidence that the ratio between the [O II]3727 and [Ne V]3426 luminosity increases with obscuration. This correlation is interpreted as evidence of enhanced star formation in obscured QSOs, which is consistent with current popular scenarios of BH-galaxy coevolution.

Acknowledgements. We thank Martin Elvis, Alessandro Marconi, Lucia Pozzetti, Guido Risaliti, Marco Salvati, and Pilar Esquej for stimulating discussions and Monica Young for kindly providing the catalog of SDSS QSOs for which XMM-Newton data were available. We thank the referee for a timely and useful report. We acknowledge partial support from ASI-INAF and PRIN/MIUR under grants I/023/05/00, I/088/06/00 and 2006-02-5203.

\section{Appendix A: Master table for the local sample}

In the tables at the end of the Appendix we present the sample of local objects. It was used to build the $\mathrm{X} / \mathrm{NeV}$ vs. X-ray absorption diagram shown in Fig. 1. The X-ray absorption, X-ray flux, and $[\mathrm{Ne} \mathrm{V}]$ flux were derived from the literature. As for X-ray data, we generally preferred to use the values obtained with the most recent and sensitive satellites, Chandra and XMM-Newton. When dealing with heavily obscured objects, though, we considered measurements obtained with Suzaku and BeppoSAX in order to map the energy range above $10 \mathrm{keV}$ and get more robust measurements of the absorbing column density. Sometimes an observed 2-10 keV flux measurement is not directly quoted in the considered literature papers: in those cases we estimated it using the published best-fit spectral parameters and/or 2-10 keV luminosity. We analyzed the archival, unpublished X-ray data of 4 objects (Mrk 78, Tol 1506.3-00, NGC 4074, IIIZw77) and reanalyzed the X-ray spectrum of NGC 1320 , since the $2-10 \mathrm{keV}$ flux we derived from the best-fit parameters in Greenhill et al. (2008) appears to differ significantly from what is listed in the 2XMM catalog. The X-ray spectra and best-fit parameters for these objects are presented below.

\section{A.1. X-ray spectra of individual objects analyzed in this work}

For the AGN observed by XMM-Newton (Mrk 78, Tol 1506.3-00, NGC 4074, NGC 1320), EPIC pn spectral results are presented, while for ASCA data (IIIZw77), we refer to SIS spectra. All of the sources presented below were pointed as targets, with the exception of NGC 4074, which was observed at an off-axis angle of $\approx 7.3^{\prime}$.

\section{A.2. XMM-Newton data}

\section{A.2.1. Mrk 78}

The X-ray spectrum of Mrk 78 (top-left panel in Fig. A.1; effective exposure time in EPIC-pn of $\approx 7.2 \mathrm{ks}$ ) is well fitted with 

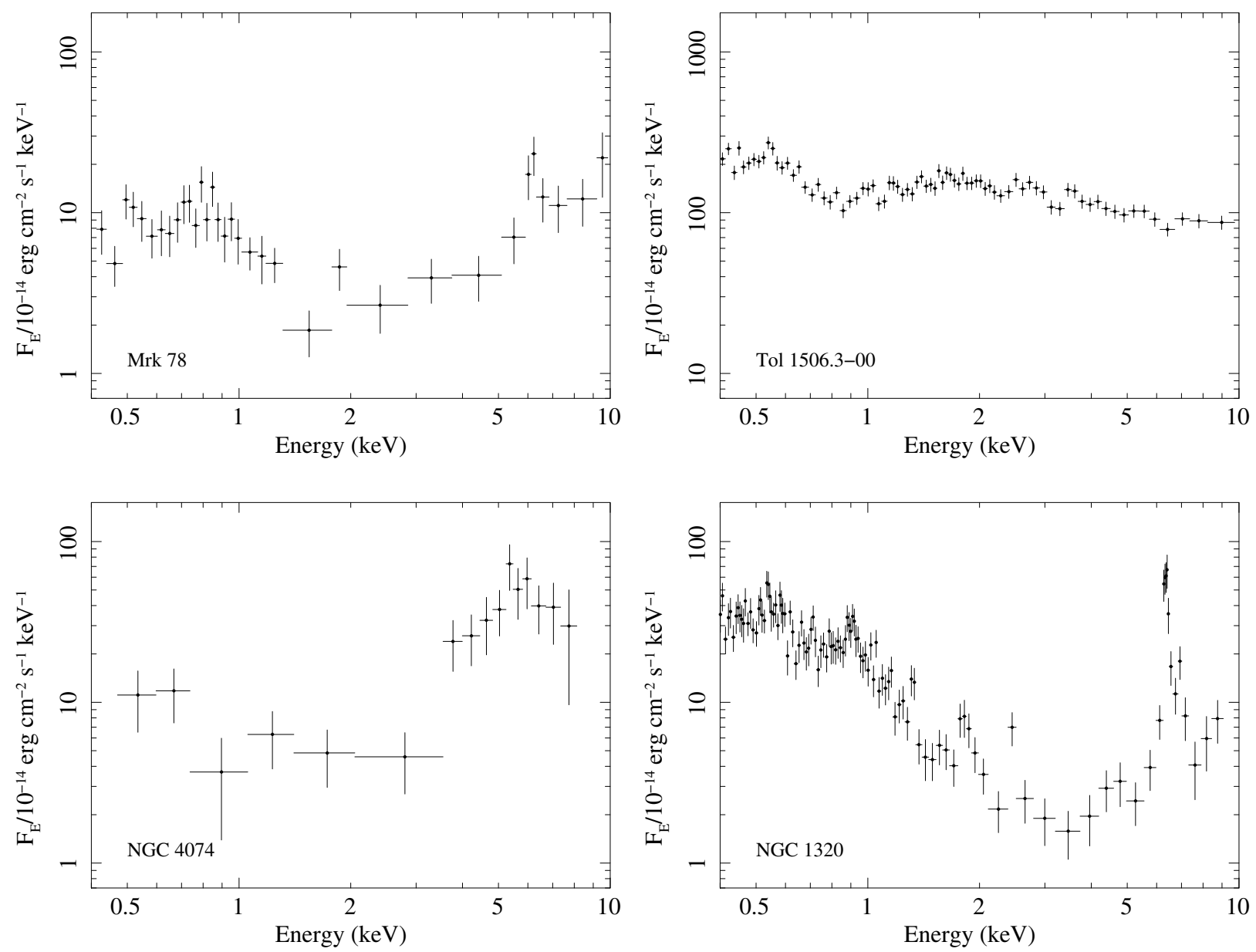

Fig. A.1. XMM-Newton EPIC-pn data of the four archival observations presented here (from top-left to bottom-right: Mrk 78, Tol 1506.3-00, NGC 4074, and NGC 1320). The data have been corrected for the effective area and further converted to flux density in units of $10^{-14} \mathrm{erg} \mathrm{cm}^{-2} \mathrm{~s}^{-1} \mathrm{keV}^{-1}$ for displaying purposes.

a model including, in the soft X-ray energy band, a thermal $(k T \approx 0.6 \mathrm{keV})$ and a power-law component (with a photon in$\operatorname{dex} \Gamma \approx 2.3$ ). The emission at energies above $2 \mathrm{keV}$ is reproduced well by an absorbed $\left(N_{\mathrm{H}} \approx 5.7 \times 10^{23} \mathrm{~cm}^{-2}\right)$ power-law (with photon index fixed to 1.8 because of the limited photon statistics) plus an iron emission line. The line energy $(E=6.31-6.45 \mathrm{keV})$ indicates emission from neutral or mildly ionized iron, while its $E W(\approx 340 \mathrm{eV})$ is consistent, within the errors, with being produced by transmission through the same matter responsible for the absorption of the nuclear component. The $0.5-2 \mathrm{keV}$ and $2-10 \mathrm{keV}$ fluxes are $\approx 8.1 \times 10^{-14} \mathrm{erg} \mathrm{cm}^{-2} \mathrm{~s}^{-1}$ and $\approx 5.9 \times$ $10^{-13} \mathrm{erg} \mathrm{cm}^{-2} \mathrm{~s}^{-1}$, respectively; the de-absorbed, rest-frame $2-10 \mathrm{keV}$ luminosity for this source is $\approx 8.5 \times 10^{42} \mathrm{erg} \mathrm{s}^{-1}$.

\section{A.2.2. Tol $1506.3-00$}

Tol 1506.3-00 was observed for $\approx 3.7 \mathrm{ks}$ with XMM-Newton (top-right panel in Fig. A.1). Its spectrum is characterized by a thermal $(k T \approx 0.2 \mathrm{keV})$ plus a power-law component at soft energies, while the emission above $\approx 2 \mathrm{keV}$ is parameterized well by a power law with $\Gamma \approx 1.6$ and mild absorption $\left(N_{\mathrm{H}} \approx\right.$ $\left.7.1 \times 10^{21} \mathrm{~cm}^{-2}\right)$. No iron line is present, the upper limit on its $E W$, in the case of neutral iron, is $\approx 40 \mathrm{eV}$ (90\% confidence level). The $0.5-2 \mathrm{keV}$ and $2-10 \mathrm{keV}$ fluxes are $\approx 2.35 \times$ $10^{-12} \mathrm{erg} \mathrm{cm}^{-2} \mathrm{~s}^{-1}$ and $\approx 7.71 \times 10^{-12} \mathrm{erg} \mathrm{cm}^{-2} \mathrm{~s}^{-1}$, respectively.
The intrinsic, rest-frame $2-10 \mathrm{keV}$ luminosity of Tol $1506.3-00$ is $\approx 5.5 \times 10^{43} \mathrm{erg} \mathrm{s}^{-1}$.

\section{A.2.3. NGC 4074}

The XMM-Newton spectrum of NGC 4074 (bottom-left panel in Fig. A.1; exposure time of $\approx 2.6 \mathrm{ks}$ ) requires a double powerlaw model, with the component at energies above $\approx 3 \mathrm{keV}$ being absorbed by a column density $\mathrm{N}_{\mathrm{H}} \approx 2.4 \times 10^{23} \mathrm{~cm}^{-2}$ (assuming $\Gamma=1.8$ ). No iron line is present, with a $90 \%$ upper limit on the $E W$ of a neutral iron line of $\approx 190 \mathrm{eV}$. The measured $0.5-2 \mathrm{keV}(2-10 \mathrm{keV})$ flux is $\approx 8.6 \times 10^{-14} \mathrm{erg} \mathrm{cm}^{-2} \mathrm{~s}^{-1}$ $\left(\approx 2.59 \times 10^{-12} \mathrm{erg} \mathrm{cm}^{-2} \mathrm{~s}^{-1}\right)$, while the de-absorbed, rest-frame $2-10 \mathrm{keV}$ luminosity is $\approx 8.0 \times 10^{42} \mathrm{erg} \mathrm{s}^{-1}$.

\section{A.2.4. NGC 1320}

Among the sources observed by XMM-Newton and presented in this Appendix, NGC 1320 is the one with the longest exposure time $(\approx 11.5 \mathrm{ks})$ and most complex X-ray spectrum, as shown in Fig. A.1 (bottom-right panel). The X-ray data for this source are highly suggestive of the presence of both a transmission and a reflection component. While the transmitted component is parameterized well by a $\Gamma=1.8$ power-law continuum absorbed by 
A\&A 519, A92 (2010)

Table A.1. The local sample: Seyfert 1.0 to 1.5, narrow line Seyfert 1s (NLS1) and broad line radio galaxies (BLRGs).

\begin{tabular}{|c|c|c|c|c|c|c|c|c|}
\hline $\begin{array}{l}\text { Name } \\
\text { (1) }\end{array}$ & $\begin{array}{l}\text { Type } \\
\text { (2) }\end{array}$ & $\begin{array}{c}z \\
(3)\end{array}$ & $\begin{array}{l}\log N_{\mathrm{H}} \\
\text { (4) }\end{array}$ & $\begin{array}{r}\log f_{2-10} \\
(5)\end{array}$ & $\begin{array}{c}\operatorname{Ref}_{X} \\
(6)\end{array}$ & $\begin{array}{r}\log f_{\mathrm{NeV}} \\
(7)\end{array}$ & $\begin{array}{c}\text { A } \\
(8)\end{array}$ & $\begin{array}{c}\operatorname{Ref}_{\mathrm{NeV}} \\
\text { (9) }\end{array}$ \\
\hline NGC 3227 & 1.5 & 0.0039 & 22.83 & -11.09 & 1 & -13.05 & $\mathrm{~L}$ & 1 \\
\hline NGC 3516 & 1.5 & 0.0088 & 20.63 & -10.62 & 2 & -13.15 & $\mathrm{~L}$ & 1 \\
\hline NGC 3783 & 1.0 & 0.0097 & 20.00 & -10.07 & 3 & -12.86 & M & 2 \\
\hline NGC 4051 & 1.5 & 0.0020 & 20.00 & -10.64 & 4 & -12.98 & $\mathrm{~L}$ & 1 \\
\hline NGC 4151 & 1.5 & 0.0033 & 22.88 & -10.35 & 1 & -11.70 & $\mathrm{~L}$ & 1 \\
\hline NGC 4253 & NLS1 & 0.0129 & 20.00 & -10.80 & 5 & -13.49 & M & 3 \\
\hline NGC 4395 & 1.0 & 0.0010 & 20.80 & -11.30 & 6 & -14.10 & $S$ & 4 \\
\hline NGC 4593 & 1.0 & 0.0090 & 20.00 & -10.43 & 7 & -13.60 & M & 2 \\
\hline NGC 5548 & 1.5 & 0.0172 & 20.00 & -10.28 & 7 & -12.57 & $\mathrm{~L}$ & 1 \\
\hline NGC 6814 & 1.5 & 0.0052 & 20.00 & -11.82 & 8 & -13.77 & M & 2 \\
\hline NGC 7469 & 1.2 & 0.0163 & 20.00 & -10.64 & 7 & -12.74 & $\mathrm{~L}$ & 1 \\
\hline Mrk 42 & NLS1 & 0.0246 & 20.00 & -11.98 & 9 & -14.52 & $\mathrm{~L}$ & 5 \\
\hline Mrk 359 & NLS1 & 0.0169 & 20.00 & -11.24 & 10 & -13.30 & $\mathrm{~S}$ & 4 \\
\hline Mrk 486 & NLS1 & 0.0389 & 20.00 & -11.55 & 11 & -13.68 & $\mathrm{~S}$ & 4 \\
\hline Mrk 493 & NLS1 & 0.0313 & 20.00 & -11.46 & 10 & -14.52 & M & 3 \\
\hline Mrk 509 & 1.2 & 0.0344 & 20.00 & -10.47 & 7 & -12.24 & M & 2 \\
\hline Mrk 704 & 1.5 & 0.0291 & 23.13 & -11.25 & 12 & -13.21 & $\mathrm{~S}$ & 4 \\
\hline Mrk 705 & 1.2 & 0.0285 & 20.00 & -10.64 & 13 & -13.80 & $\mathrm{~S}$ & 4 \\
\hline Mrk 841 & 1.5 & 0.0364 & 20.00 & -10.79 & 14 & -13.15 & M & 2 \\
\hline Mrk 896 & 1.0 & 0.0264 & 20.00 & -11.46 & 10 & -14.10 & $\mathrm{M}$ & 2 \\
\hline Mrk 926 & 1.5 & 0.0469 & 20.00 & -10.51 & 7 & -13.96 & S & 6 \\
\hline Mrk 1239 & NLS1 & 0.0194 & 23.52 & -11.82 & 15 & -13.31 & $\mathrm{~S}$ & 4 \\
\hline $3 \mathrm{C} 120$ & BLRG & 0.0330 & 20.70 & -10.31 & 16 & -13.72 & $\mathrm{~S}$ & 6 \\
\hline $3 \mathrm{C} 227$ & BLRG & 0.0858 & 22.10 & -11.70 & 17 & -14.40 & M & 7 \\
\hline 3C 382 & BLRG & 0.0578 & 20.00 & -10.21 & 18 & -13.85 & M & 7 \\
\hline 3 C 390.3 & BLRG & 0.0561 & 20.60 & -10.40 & 19 & -13.59 & $S$ & 4 \\
\hline $3 \mathrm{C} 445$ & BLRG & 0.0562 & 22.70 & -11.17 & 20 & -13.85 & $\mathrm{M}$ & 2 \\
\hline Fairall 9 & 1.2 & 0.0470 & 20.00 & -10.92 & 7 & -13.03 & M & 8 \\
\hline Fairall 51 & 1.0 & 0.0142 & 22.20 & -10.60 & 21 & -13.29 & M & 2 \\
\hline Fairall 1116 & 1.0 & 0.0582 & 20.00 & -11.27 & 22 & -13.54 & M & 8 \\
\hline Tol 1351-375 & 1.9 & 0.0520 & 22.20 & -11.42 & 23 & -13.48 & $\mathrm{M}$ & 2 \\
\hline Tol 1506.3-00 & 1.5 & 0.0543 & 21.85 & -11.11 & 24 & -14.00 & M & 2 \\
\hline H1143-182 & 1.5 & 0.0329 & 20.00 & -10.55 & 4 & -13.19 & $\mathrm{M}$ & 8 \\
\hline H1846-786 & 1.0 & 0.0743 & 20.00 & -11.10 & 7 & -13.49 & M & 2 \\
\hline Akn 120 & 1.0 & 0.0330 & 20.00 & -10.42 & 25 & -14.15 & $\mathrm{~S}$ & 4 \\
\hline Akn 564 & NLS1 & 0.0247 & 20.00 & -10.80 & 26 & -13.33 & $S$ & 4 \\
\hline MCG-6-30-15 & 1.2 & 0.0077 & 20.00 & -10.62 & 27 & -14.05 & M & 2 \\
\hline 1ES1615+061 & 1.5 & 0.0380 & 20.00 & -11.10 & 28 & -14.22 & M & 2 \\
\hline IIIZw77 & 1.2 & 0.0342 & 23.90 & -12.22 & 24 & -13.55 & $\mathrm{M}$ & 9 \\
\hline ESO 141-G55 & 1.2 & 0.0360 & 20.00 & -10.57 & 29 & -13.17 & $\mathrm{M}$ & 8 \\
\hline POX 52 & 1.0 & 0.0218 & 20.70 & -12.11 & 30 & -13.35 & M & 10 \\
\hline
\end{tabular}

Notes. Column description: (1) Source name. (2) Spectroscopic classification (1.0, 1.2 and 1.5 refer to Seyfert types). (3) Redshift. (4) Logarithm of the cold X-ray absorbing column density. For those objects in which no cold absorption is measured in excess of the Galactic value, the column has been fixed to $\log N_{\mathrm{H}}=20$. (5) Logarithm of the observed 2-10 keV flux in erg cm $\mathrm{cm}^{-2} \mathrm{~s}^{-1}$. (6) Reference for the X-ray data. (7) Logarithm of the $[\mathrm{Ne} \mathrm{V}] 3426$ flux in erg cm $\mathrm{cm}^{-2} \mathrm{~s}^{-1}$. (8) Aperture used to measure the [Ne V] flux, defined following Schmitt (1998): S (small), M (medium) and $\mathrm{L}$ (large) correspond to apertures in the ranges 1-3", 3-7", and $>7^{\prime \prime}$, respectively. (9) Reference for the [Ne V] data.

X-ray references: (1) Cappi et al. (2006); (2) Bianchi et al. (2004); (3) Blustin et al. (2002); (4) Bianchi et al. (2009); (5) Landi et al. (2005); (6) Iwasawa et al. (2010); (7) Shinozaki et al. (2006); (8) Vasudevan \& Fabian (2007); (9) Vaughan et al. (1999); (10) Gallo (2006); (11) Ballo et al. (2008); (12) Landi et al. (2007); (13) Gallo et al. (2005); (14) Piconcelli et al. (2005); (15) Grupe et al. (2004); (16) Grandi et al. (2006); (17) Hardcastle et al. (2007); (18) Gliozzi et al. (2007); (19) Evans et al. (2006); (20) Sambruna et al. (2007); (21) Jiménez-Bailón et al. (2008) (22) D'Ammando et al. (2008); (23) Risaliti et al. (2000); (24) this work; (25) Vaughan et al. (2004); (26) Vignali et al. (2004); (27) Ponti et al. (2004); (28) Guainazzi et al. (1998); (29) Gondoin et al. (2003); (30) Thornton et al. (2008).

[Ne V] references: (1) Anderson (1970); (2) Morris \& Ward (1988); (3) Osterbrock \& Pogge (1985); (4) Erkens et al. (1997); (5) Malkan (1986); (6) Durret \& Bergeron (1988); (7) Osterbrock et al. (1976); (8) Winkler (1992); (9) Osterbrock (1981b); (10) Kunth et al. (1987).

thick matter (with a column density of $\approx 2.4 \times 10^{24} \mathrm{~cm}^{-2}$ ), the parameters are basically unconstrained for the reflection component, calling for observations with higher photon statistics and data above $10 \mathrm{keV}$ to properly model and constrain the two spectral components. The best-fitting model requires also the presence of a power-law $(\Gamma \approx 2.9)$ at low energies and a $\mathrm{Ne}$ IX emission line at an energy of $\approx 930 \mathrm{eV}(E W \approx 50 \mathrm{eV})$. The measured $0.5-2 \mathrm{keV}(2-10 \mathrm{keV})$ flux is $\approx 2.3 \times$ $10^{-13} \mathrm{erg} \mathrm{cm} \mathrm{cm}^{-1}\left(\approx 5.7 \times 10^{-13} \mathrm{erg} \mathrm{cm}^{-2} \mathrm{~s}^{-1}\right)^{8}$, and the de-absorbed, rest-frame $2-10 \mathrm{keV}$ luminosity is $\approx 8.4 \times$ $10^{42} \mathrm{erg} \mathrm{s}^{-1}$; however, we note that this value is somehow uncertain, given the complex X-ray modeling of the data presented here.

\footnotetext{
${ }^{8}$ Consistent with the value quoted in the 2 XMM catalog.
} 
R. Gilli et al.: [Ne V] selected obscured QSOs

Table A.2. The local sample: Seyfert 1.8 to 2.0 and narrow line radio galaxies (NLRGs).

\begin{tabular}{|c|c|c|c|c|c|c|c|c|}
\hline $\begin{array}{l}\text { Name } \\
\text { (1) }\end{array}$ & $\begin{array}{l}\text { Type } \\
(2)\end{array}$ & $\begin{array}{c}z \\
(3)\end{array}$ & $\begin{array}{c}\log N_{\mathrm{H}} \\
\text { (4) }\end{array}$ & $\begin{array}{r}f_{2-10} \\
(5)\end{array}$ & $\begin{array}{c}\operatorname{Ref}_{X} \\
(6)\end{array}$ & $\begin{array}{r}f_{\mathrm{NeV}} \\
(7)\end{array}$ & $\begin{array}{c}\text { A } \\
(8)\end{array}$ & $\begin{array}{c}\operatorname{Ref}_{\mathrm{NeV}} \\
\text { (9) }\end{array}$ \\
\hline NGC 1068 & 2.0 & 0.0038 & 25.00 & -11.34 & 1 & -11.94 & $\mathrm{M}$ & 1 \\
\hline NGC 1275 & NLRG & 0.0175 & 20.00 & -10.91 & $2^{a}$ & -12.70 & $\mathrm{~L}$ & 2 \\
\hline NGC 1320 & 2.0 & 0.0089 & 24.00 & -12.24 & 3 & -13.15 & S & 3 \\
\hline NGC 1667 & 2.0 & 0.0152 & 24.00 & -13.00 & 4 & -14.00 & $\mathrm{~L}$ & 4 \\
\hline NGC 2992 & 1.9 & 0.0077 & 21.95 & -10.13 & 5 & -14.52 & S & 5 \\
\hline NGC 3081 & 2.0 & 0.0079 & 23.85 & -11.89 & 6 & -12.66 & $\mathrm{~L}$ & 6 \\
\hline NGC 3281 & 2.0 & 0.0106 & 24.18 & -11.54 & 7 & -14.52 & $S$ & 5 \\
\hline NGC 3393 & 2.0 & 0.0125 & 25.00 & -12.68 & 8 & -13.25 & $\mathrm{~L}$ & 4 \\
\hline NGC 4074 & 2.0 & 0.0224 & 23.38 & -11.59 & 3 & -14.05 & $S$ & 7 \\
\hline NGC 4507 & 2.0 & 0.0118 & 23.63 & -10.89 & 9 & -13.26 & $\mathrm{~L}$ & 6 \\
\hline NGC 5135 & 2.0 & 0.0136 & 24.00 & -12.80 & 10 & -13.54 & $\mathrm{~L}$ & 4 \\
\hline NGC 5506 & 1.9 & 0.0062 & 22.50 & -10.10 & 11 & -13.96 & $\mathrm{M}$ & 8 \\
\hline NGC 5643 & 2.0 & 0.0040 & 23.80 & -12.08 & 12 & -13.33 & M & 8 \\
\hline NGC 5728 & 2.0 & 0.0093 & 24.30 & -11.89 & 13 & -13.66 & $\mathrm{~L}$ & 4 \\
\hline NGC 7130 & 2.0 & 0.0161 & 24.00 & -12.29 & 14 & -14.00 & $\mathrm{~S}$ & 9 \\
\hline NGC 7314 & 1.9 & 0.0048 & 22.02 & -10.39 & 15 & -14.30 & $\mathrm{M}$ & 8 \\
\hline NGC 7582 & 2.0 & 0.0052 & 24.00 & -11.64 & 16 & -12.48 & M & 10 \\
\hline NGC 7674 & 2.0 & 0.0289 & 25.00 & -12.12 & 17 & -13.40 & $\mathrm{~L}$ & 11 \\
\hline Mrk 1 & 2.0 & 0.0159 & 24.00 & -12.89 & 18 & -13.49 & $\mathrm{~L}$ & 11 \\
\hline Mrk 34 & 2.0 & 0.0505 & 24.00 & -12.74 & 19 & -13.55 & $\mathrm{~L}$ & 11 \\
\hline Mrk 78 & 2.0 & 0.0371 & 23.76 & -12.23 & 3 & -13.85 & $\mathrm{~L}$ & 11 \\
\hline Mrk 348 & 2.0 & 0.0150 & 23.13 & -10.56 & 20 & -13.64 & $\mathrm{~L}$ & 11 \\
\hline Mrk 463E & 2.0 & 0.0500 & 23.85 & -12.39 & 21 & -13.89 & $\mathrm{~L}$ & 11 \\
\hline Mrk 477 & 2.0 & 0.0377 & 23.38 & -11.92 & 22 & -13.14 & $\mathrm{~L}$ & 4 \\
\hline Mrk 573 & 2.0 & 0.0171 & 24.00 & -12.92 & 23 & -13.00 & $\mathrm{~S}$ & 3 \\
\hline Mrk 609 & 1.8 & 0.0344 & 20.00 & -11.83 & 24 & -14.40 & S & 12 \\
\hline Mrk 612 & 2.0 & 0.0204 & 23.81 & -12.44 & 18 & -13.66 & $\mathrm{~L}$ & 11 \\
\hline $3 \mathrm{C} 33$ & NLRG & 0.0597 & 23.59 & -11.13 & 25 & -14.70 & M & 1 \\
\hline MCG-5-23-16 & 2.0 & 0.0085 & 22.15 & -10.10 & 26 & -13.89 & $S$ & 5 \\
\hline WAS 49b & 2.0 & 0.0630 & 22.80 & -12.20 & 20 & -14.30 & $S$ & 13 \\
\hline Cygnus A & NLRG & 0.0560 & 23.23 & -10.89 & 25 & -14.40 & M & 14 \\
\hline IC 3639 & 2.0 & 0.0109 & 24.00 & -13.10 & 18 & -13.60 & $\mathrm{~L}$ & 4 \\
\hline Tol 0109-383 & 2.0 & 0.0117 & 24.30 & -11.80 & 27 & -13.62 & S & 15 \\
\hline
\end{tabular}

Notes. Column description: (1) Source name. (2) Spectroscopic classification (1.8, 1.9 and 2.0 refer to Seyfert types). (3) Redshift. (4) Logarithm of the cold X-ray absorbing column density. For those objects in which no cold absorption is measured in excess of the Galactic value, the column has been fixed to $\log N_{\mathrm{H}}=20$. (5) Observed 2-10 keV flux in units of $\mathrm{erg} \mathrm{cm}^{-2} \mathrm{~s}^{-1}$. (6) Reference for the X-ray data. (7) [Ne V]3426 flux in units of erg cm $\mathrm{cm}^{-2} \mathrm{~s}^{-1}$. (8) Aperture used to measure the [Ne V] flux, defined following Schmitt (1998): S (small), M (medium) and L (large) correspond to apertures in the ranges $1-3^{\prime \prime}, 3-7 "$, and $>7$ ", respectively. (9) Reference for the [Ne V] data.

$X$-ray references: (1) Cappi et al. (2006); (2) Panessa et al. (2006); (3) this work; (4) Bianchi et al. (2005); (5) Gilli et al. (2000); (6) Bassani et al. (1999); (7) Vignali \& Comastri (2002); (8) Maiolino et al. (1998); (9) Matt et al. (2004); (10) Guainazzi et al. (2005a); (11) Bianchi et al. (2003); (12) Guainazzi et al. (2004); (13) Comastri et al. (2010); (14) Risaliti et al. (1999); (15) Dewangan \& Griffiths (2005); (16) Piconcelli et al. (2007a); (17) Malaguti et al. (1998); (18) Guainazzi et al. (2005b); (19) Greenhill et al. (2008); (20) Awaki et al. (2000); (21) Bianchi et al. (2008); (22) Levenson et al. (2001); (23) Shu et al. (2007); (24) Gallo et al. (2006); (25) Evans et al. (2006); (26) Balestra et al. (2004); (27) Iwasawa et al. (2001b)

(a) The column density of NGC 1275, which is at the center within the Perseus cluster, is difficult to measure: Evans et al. (2006) do not find absorption in excess of the Galactic one based on Chandra data.

[Ne V] references: (1) Koski (1978); (2) Anderson (1970); (3) Erkens et al. 1997; (4) This work (based on the catalog of UV-optical spectra of nearby galaxies by Storchi-Bergmann et al., see text); (5) Durret \& Bergeron (1988); (6) Durret \& Bergeron (1986); (7) Shuder \& Osterbrock (1981); (8) Morris \& Ward (1988); (9) Shields \& Filippenko (1990); (10) Ward et al. (1980); (11) Malkan (1986); (12) Osterbrock (1981a); (13) Moran et al. (1992); (14) Osterbrock \& Miller (1975); (15) Fosbury \& Sansom (1983).

\section{A.3. ASCA data}

\section{A.3.1. IIIZw77}

An ASCA observation with a net exposure time of $\approx 65 \mathrm{ks}$ has detected a faint X-ray source in this galaxy. The X-ray spectrum (see Fig. A.2) shows a hard X-ray excess above $3 \mathrm{keV}$ when a simple power law is fitted. This hard X-ray component probably originates in an obscured active nucleus. When modeled by an absorbed power law with photon index $\Gamma=1.8$, the absorbing column density is estimated to be $N_{\mathrm{H}}=8_{-4}^{+5} \times 10^{23} \mathrm{~cm}^{-2}$. The data is not good enough to constrain an Fe $\mathrm{K}$ emissionline. The soft X-ray component can be described by a power law of $\Gamma \approx 2.5$. While the origin of the soft X-ray emission is unclear, it is likely extended photoionized gas, assuming that no strong star formation is taking place in this E/S0 galaxy. The 
A\&A 519, A92 (2010)

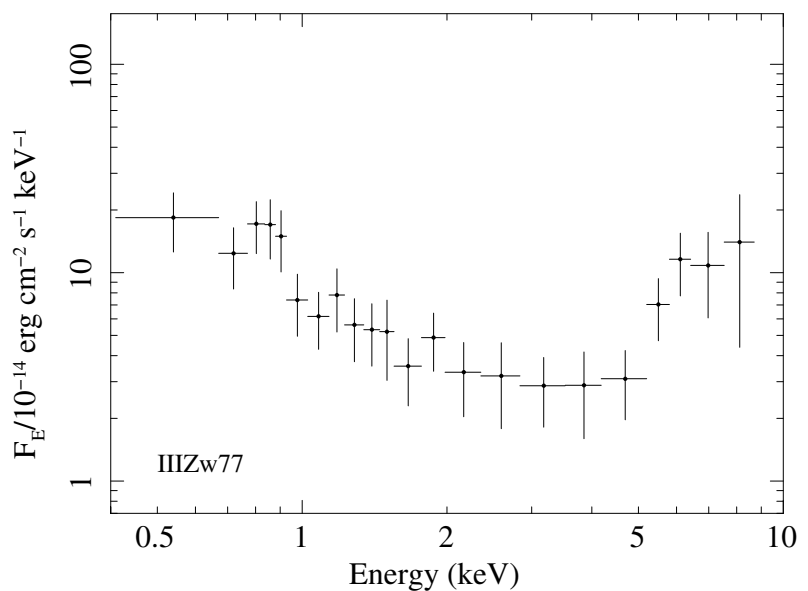

Fig. A.2. ASCA data of the source IIIZw77, corrected for the effective area and further converted to flux density in units of $10^{-14} \mathrm{erg} \mathrm{cm}^{-2} \mathrm{~s}^{-1} \mathrm{keV}^{-1}$ for displaying purposes.

observed fluxes in the $0.5-2 \mathrm{keV}$ and $2-10 \mathrm{keV}$ bands are $1 \times$ $10^{-13} \mathrm{erg} \mathrm{cm}^{-2} \mathrm{~s}^{-1}$ and $6 \times 10^{-13} \mathrm{erg} \mathrm{cm}^{-2} \mathrm{~s}^{-1}$, respectively. The absorption-corrected $2-10 \mathrm{keV}$ luminosity derived from the absorbed power-law model is $1 \times 10^{43} \mathrm{erg} \mathrm{s}^{-1}$.

\section{References}

Alexander, D. M., Smail, I., Bauer, F. E., et al. 2005, Nature, 434, 738 Alexander, D. M., Chary, R., Pope, A., et al. 2008, ApJ, 687, 835 Anderson, K. S. 1970, ApJ, 162, 743

Awaki, H., Ueno, S., Taniguchi, Y., \& Weaver, K. A. 2000, ApJ, 542, 175

Balestra, I., Bianchi, S., \& Matt, G. 2004, A\&A, 415, 437

Ballantyne, D. R., Everett, J. E., \& Murray, N. 2006, ApJ, 639, 740

Ballo, L., Giustini, M., Schartel, N., et al. 2008, A\&A, 483, 137

Barth, A. J., Ho, L. C., Rutledge, R. E., \& Sargent, W. L. W. 2004, ApJ, 607, 90

Bassani, L., Dadina, M., Maiolino, R., et al. 1999, ApJS, 121, 473

Bianchi, S., Balestra, I., Matt, G., Guainazzi, M., \& Perola, G. C. 2003, A\&A, 402, 141

Bianchi, S., Matt, G., Balestra, I., Guainazzi, M., \& Perola, G. C. 2004, A\&A, 422, 65

Bianchi, S., Guainazzi, M., Matt, G., et al. 2005, A\&A, 442, 185

Bianchi, S., Guainazzi, M., \& Chiaberge, M. 2006, A\&A, 448, 499

Bianchi, S., Chiaberge, M., Piconcelli, E., Guainazzi, M., \& Matt, G. 2008, MNRAS, 386, 105

Bianchi, S., Guainazzi, M., Matt, G., Fonseca Bonilla, N., \& Ponti, G. 2009, A\&A, 495, 421

Blustin, A. J., Branduardi-Raymont, G., Behar, E., et al. 2002, A\&A, 392, 453

Bongiorno, A., Mignoli, M., Zamorani, G., et al. 2010, A\&A, 510, A56

Brandt, W. N., \& Hasinger, G. 2005, ARA\&A, 43, 827

Cappi, M., Panessa, F., Bassani, L., et al. 2006, A\&A, 446, 459

Cash, W. 1979, ApJ, 228, 939

Colless, M., Dalton, G., Maddox, S., et al. 2001, MNRAS, 328, 1039

Comastri, A. 2004, in Supermassive Black Holes in the Distant Universe, ed. A. J. Barger, Astrophys. Space Sci. Libr., 308, 245

Comastri, A., Iwasawa, K., Gilli, R., et al. 2010, ApJ, 717, 787

Corbett, E. A., Croom, S. M., Boyle, B. J., et al. 2003, MNRAS, 343, 705

Daddi, E., Alexander, D. M., Dickinson, M., et al. 2007, ApJ, 670, 173

Dahari, O., \& De Robertis, M. M. 1988, ApJ, 331, 727

D’Ammando, F., Bianchi, S., Jiménez-Bailón, E., \& Matt, G. 2008, A\&A, 482, 499

Della Ceca, R., Severgnini, P., Caccianiga, A., et al. 2008, Mem. Soc. Astron. Ital., 79, 65

Dewangan, G. C., \& Griffiths, R. E. 2005, ApJ, 625, L31

Diamond-Stanic, A. M., Rieke, G. H., \& Rigby, J. R. 2009, ApJ, 698, 623

Dickey, J. M., \& Lockman, F. J. 1990, ARA\&A, 28, 215

Durret, F., \& Bergeron, J. 1986, A\&A, 156, 51

Durret, F., \& Bergeron, J. 1988, A\&AS, 75, 273

Elvis, M., Civano, F., Vignali, C., et al. 2009, ApJS, 184, 158

Erkens, U., Appenzeller, I., \& Wagner, S. 1997, A\&A, 323, 707

Evans, D. A., Worrall, D. M., Hardcastle, M. J., Kraft, R. P., \& Birkinshaw, M. 2006, ApJ, 642, 96
Ferland, G. J., \& Osterbrock, D. E. 1986, ApJ, 300, 658

Fiore, F., Grazian, A., Santini, P., et al. 2008, ApJ, 672, 94

Fiore, F., Puccetti, S., Brusa, M., et al. 2009, ApJ, 693, 447

Fosbury, R. A. E., \& Sansom, A. E. 1983, MNRAS, 204, 1231

Franceschini, A., Bassani, L., Cappi, M., et al. 2000, A\&A, 353, 910

Gallo, L. C. 2006, MNRAS, 368, 479

Gallo, L. C., Balestra, I., Costantini, E., et al. 2005, A\&A, 442, 909

Gallo, L. C., Lehmann, I., Pietsch, W., et al. 2006, MNRAS, 365, 688

Gaskell, C. M., \& Benker, A. J. 2007, unpublished [arXiv: 0711. 1013]

Georgantopoulos, I., Georgakakis, A., \& Akylas, A. 2007, A\&A, 466, 823

Georgantopoulos, I., Akylas, A., Georgakakis, A., \& Rowan-Robinson, M. 2009,

A\&A, 507, 747

Gilli, R., Maiolino, R., Marconi, A., et al. 2000, A\&A, 355, 485

Gilli, R., Comastri, A., \& Hasinger, G. 2007, A\&A, 463, 79

Gliozzi, M., Sambruna, R. M., Eracleous, M., \& Yaqoob, T. 2007, ApJ, 664, 88

Gondoin, P., Orr, A., \& Lumb, D. 2003, A\&A, 398, 967

Grandi, P., Malaguti, G., \& Fiocchi, M. 2006, ApJ, 642, 113

Greenhill, L. J., Tilak, A., \& Madejski, G. 2008, ApJ, 686, L13

Grupe, D., Mathur, S., \& Komossa, S. 2004, AJ, 127, 3161

Guainazzi, M., Piro, L., Capalbi, M., et al. 1998, A\&A, 339, 337

Guainazzi, M., Rodriguez-Pascual, P., Fabian, A. C., Iwasawa, K., \& Matt, G. 2004, MNRAS, 355, 297

Guainazzi, M., Fabian, A. C., Iwasawa, K., Matt, G., \& Fiore, F. 2005a, MNRAS, 356,295

Guainazzi, M., Matt, G., \& Perola, G. C. 2005b, A\&A, 444, 119

Hardcastle, M. J., Croston, J. H., \& Kraft, R. P. 2007, ApJ, 669, 893

Hopkins, P. F., Hernquist, L., Cox, T. J., et al. 2006, ApJS, 163, 1

Hopkins, P. F., Hernquist, L., Cox, T. J., \& Kereš, D. 2008, ApJS, 175, 356

Iwasawa, K., Fabian, A. C., \& Ettori, S. 2001a, MNRAS, 321, L15

Iwasawa, K., Matt, G., Fabian, A. C., et al. 2001b, MNRAS, 326, 119

Iwasawa, K., Tanaka, Y., \& Gallo, L. C. 2010, A\&A, 514, A58

Jiménez-Bailón, E., Guainazzi, M., Matt, G., et al. 2008, in Rev. Mex. Astron. Astrofis. Conf. Ser., 32, 131

Kauffmann, G., \& Haehnelt, M. 2000, MNRAS, 311, 576

Kewley, L. J., Geller, M. J., \& Jansen, R. A. 2004, AJ, 127, 2002

Kim, M., Ho, L. C., \& Im, M. 2006, ApJ, 642, 702

Koski, A. T. 1978, ApJ, 223, 56

Kunth, D., Sargent, W. L. W., \& Bothun, G. D. 1987, AJ, 93, 29

Lamastra, A., Bianchi, S., Matt, G., et al. 2009, A\&A, 504, 73

Landi, R., Malizia, A., \& Bassani, L. 2005, A\&A, 441, 69

Landi, R., Masetti, N., Morelli, L., et al. 2007, ApJ, 669, 109

Lavalley, M., Isobe, T., \& Feigelson, E. 1992, in Astronomical Data Analysis Software and Systems I, ed. D. M. Worrall, C. Biemesderfer, \& J. Barnes, ASP Conf. Ser., 25, 245

Levenson, N. A., Weaver, K. A., \& Heckman, T. M. 2001, ApJ, 550, 230

Lilly, S. J., Le Fèvre, O., Renzini, A., et al. 2007, ApJS, 172, 70

Lilly, S. J., LeBrun, V., Maier, C., et al. 2009, ApJS, 184, 218

Maiolino, R., Salvati, M., Bassani, L., et al. 1998, A\&A, 338, 781

Malaguti, G., Palumbo, G. G. C., Cappi, M., et al. 1998, A\&A, 331, 519

Malizia, A., Stephen, J. B., Bassani, L., et al. 2009, MNRAS, 399, 944

Malkan, M. A. 1986, ApJ, 310, 679

Marconi, A., Risaliti, G., Gilli, R., et al. 2004, MNRAS, 351, 169

Martínez-Sansigre, A., Rawlings, S., Lacy, M., et al. 2005, Nature, 436, 666

Marulli, F., Bonoli, S., Branchini, E., Moscardini, L., \& Springel, V. 2008, MNRAS, 385, 1846

Matt, G., Bianchi, S., D’Ammando, F., \& Martocchia, A. 2004, A\&A, 421, 473

Moran, E. C., Halpern, J. P., Bothun, G. D., \& Becker, R. H. 1992, AJ, 104, 990

Morris, S. L., \& Ward, M. J. 1988, MNRAS, 230, 639

Murphy, K. D., Yaqoob, T., \& Terashima, Y. 2007, ApJ, 666, 96

Osterbrock, D. E. 1981a, ApJ, 249, 462

Osterbrock, D. E. 1981b, ApJ, 246, 696

Osterbrock, D. E., \& Miller, J. S. 1975, ApJ, 197, 535

Osterbrock, D. E., \& Pogge, R. W. 1985, ApJ, 297, 166

Osterbrock, D. E., Koski, A. T., \& Phillips, M. M. 1976, ApJ, 206, 898

Panessa, F., Bassani, L., Cappi, M., et al. 2006, A\&A, 455, 173

Piconcelli, E., Jimenez-Bailón, E., Guainazzi, M., et al. 2005, A\&A, 432, 15

Piconcelli, E., Bianchi, S., Guainazzi, M., Fiore, F., \& Chiaberge, M. 2007a, A\&A, 466, 855

Piconcelli, E., Fiore, F., Nicastro, F., et al. 2007b, A\&A, 473, 85

Ponti, G., Cappi, M., Dadina, M., \& Malaguti, G. 2004, A\&A, 417, 451

Ptak, A., Zakamska, N. L., Strauss, M. A., et al. 2006, ApJ, 637, 147

Puccetti, S., Vignali, C., Cappelluti, N., et al. 2009, ApJS, 185, 586

Reyes, R., Zakamska, N. L., Strauss, M. A., et al. 2008, AJ, 136, 2373

Rigby, J. R., Diamond-Stanic, A. M., \& Aniano, G. 2009, ApJ, 700, 1878

Risaliti, G., Maiolino, R., \& Salvati, M. 1999, ApJ, 522, 157

Risaliti, G., Gilli, R., Maiolino, R., \& Salvati, M. 2000, A\&A, 357, 13

Sambruna, R. M., Reeves, J. N., \& Braito, V. 2007, ApJ, 665, 1030

Schmidt, M., Hasinger, G., Gunn, J., et al. 1998, A\&A, 329, 495 
R. Gilli et al.: [Ne V] selected obscured QSOs

Schmitt, H. R. 1998, ApJ, 506, 647

Scoville, N., Aussel, H., Brusa, M., et al. 2007, ApJS, 172, 1

Shankar, F., Salucci, P., Granato, G. L., De Zotti, G., \& Danese, L. 2004, MNRAS, 354, 1020

Shields, J. C., \& Filippenko, A. V. 1990, AJ, 100, 1034

Shinozaki, K., Miyaji, T., Ishisaki, Y., Ueda, Y., \& Ogasaka, Y. 2006, AJ, 131, 2843

Shu, X. W., Wang, J. X., Jiang, P., Fan, L. L., \& Wang, T. G. 2007, ApJ, 657, 167

Shuder, J. M., \& Osterbrock, D. E. 1981, ApJ, 250, 55

Silverman, J. D., Lamareille, F., Maier, C., et al. 2009, ApJ, 696, 396

Spinelli, P. F., Storchi-Bergmann, T., Brandt, C. H., \& Calzetti, D. 2006, ApJS, 166,498

Storchi-Bergmann, T., Kinney, A. L., \& Challis, P. 1995, ApJS, 98, 103

Thornton, C. E., Barth, A. J., Ho, L. C., Rutledge, R. E., \& Greene, J. E. 2008, ApJ, 686, 892

Tozzi, P., Gilli, R., Mainieri, V., et al. 2006, A\&A, 451, 457

Treister, E., Cardamone, C. N., Schawinski, K., et al. 2009a, ApJ, 706, 535

Treister, E., Urry, C. M., \& Virani, S. 2009b, ApJ, 696, 110
Tueller, J., Mushotzky, R. F., Barthelmy, S., et al. 2008, ApJ, 681, 113 Urrutia, T., Lacy, M., Gregg, M. D., \& Becker, R. H. 2005, ApJ, 627, 75 Vanden Berk, D. E., Richards, G. T., Bauer, A., et al. 2001, AJ, 122, 549 Vasudevan, R. V., \& Fabian, A. C. 2007, MNRAS, 381, 1235

Vaughan, S., Reeves, J., Warwick, R., \& Edelson, R. 1999, MNRAS, 309, 113 Vaughan, S., Fabian, A. C., Ballantyne, D. R., et al. 2004, MNRAS, 351, 193 Vignali, C., \& Comastri, A. 2002, A\&A, 381, 834

Vignali, C., Brandt, W. N., Boller, T., Fabian, A. C., \& Vaughan, S. 2004, MNRAS, 347, 854

Vignali, C., Alexander, D. M., \& Comastri, A. 2006, MNRAS, 373, 321 Vignali, C., Alexander, D. M., Gilli, R., \& Pozzi, F. 2010, MNRAS, 404, 48 (V10)

Ward, M., Penston, M. V., Blades, J. C., \& Turtle, A. J. 1980, MNRAS, 193, 563 Wilkes, B. J., Schmidt, G. D., Cutri, R. M., et al. 2002, ApJ, 564, L65

Wilkes, B. J., Pounds, K. A., Schmidt, G. D., et al. 2005, ApJ, 634, 183 Winkler, H. 1992, MNRAS, 257, 677

Young, M., Elvis, M., \& Risaliti, G. 2009a, ApJS, 183, 17 (Y09)

Young, M., Elvis, M., \& Risaliti, G. 2009b, ApJS, 185, 250

Zakamska, N. L., Strauss, M. A., Krolik, J. H., et al. 2003, AJ, 126, 2125 